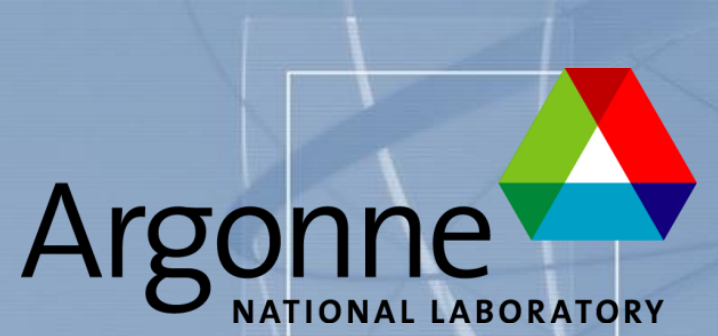

ANL/ESD/12-14

\title{
Computational Mechanics
}

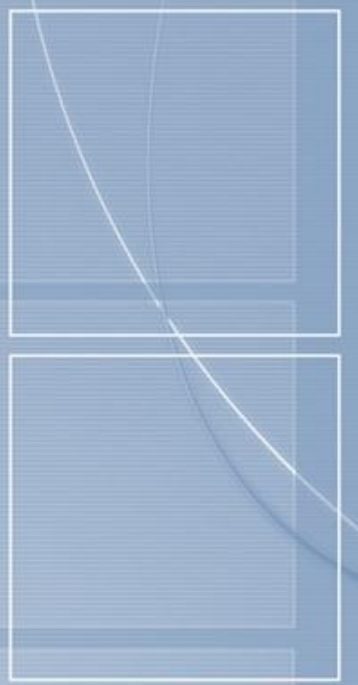

Research and Support for Aerodynamics and Hydraulics at TFHRC

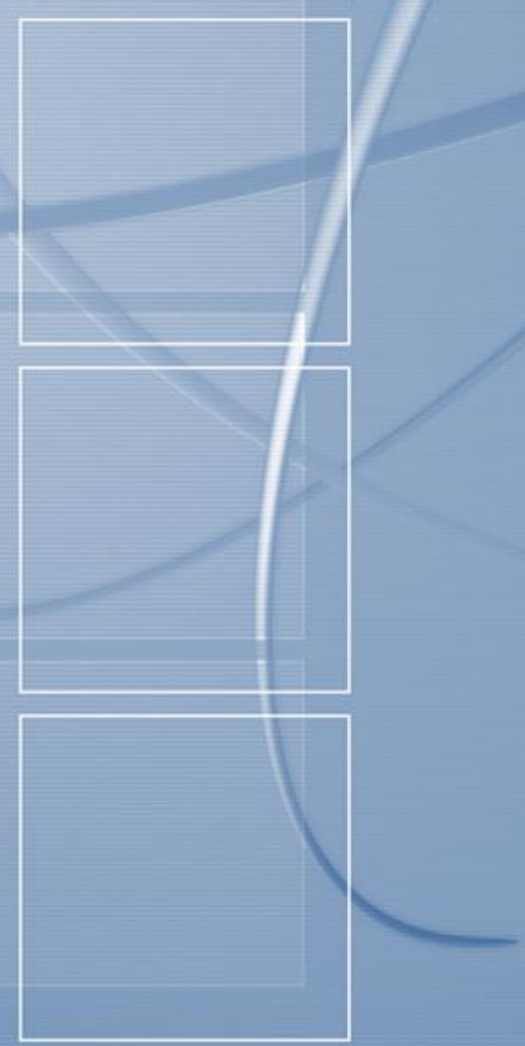

Year 2 Quarter 4 Progress Report 
About Argonne National Laboratory

Argonne is a U.S. Department of Energy laboratory managed by UChicago Argonne, LLC under contract DE-AC02-06CH11357. The Laboratory's main facility is outside Chicago, at 9700 South Cass Avenue, Argonne, Illinois 60439. For information about Argonne and its pioneering science and technology programs, see www.anl.gov.

\section{Availability of This Report}

This report is available, at no cost, at http://www.osti.gov/bridge. It is also available

on paper to the U.S. Department of Energy and its contractors, for a processing fee, from:

U.S. Department of Energy

Office of Scientific and Technical Information

P.O. Box 62

Oak Ridge, TN 37831-0062

phone (865) 576-8401

fax (865) 576-5728

reports@adonis.osti.gov information, apparatus, product, or process disclosed, or represents that its use would not infringe privately owned rights. Reference herein to any specific commercial product, process, or service by trade name, trademark, manufacturer, or otherwise, does not necessarily constitute or imply its endorsement, recommendation, or favoring by the United States Government or any agency thereof. The views and opinions of document authors expressed herein do not necessarily state or reflect those of the United States Government or any agency thereof, Argonne National Laboratory, or UChicago Argonne, LLC. 


\section{Computational Mechanics Research and Support for Aerodynamics and Hydraulics at TFHRC, Year 2 Quarter 4 Progress Report}

by

S.A. Lottes ${ }^{1}$, C. Bojanowski ${ }^{1}$, J. Shen ${ }^{2}$, and Z. Xie ${ }^{2}$

${ }^{1}$ Transportation Research and Analysis Computing Center (TRACC)

Energy Systems Division, Argonne National Laboratory

${ }^{2}$ Turner-Fairbank Highway Research Center

submitted to

Kornel Kerenyi ${ }^{1}$ and Harold Bosch ${ }^{1}$

${ }^{1}$ Turner-Fairbank Highway Research Center

November 2012 


\section{Table of Contents}

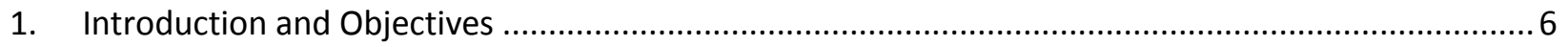

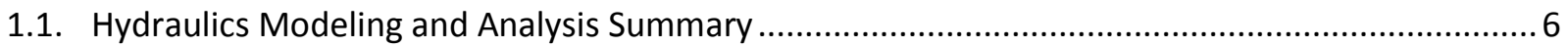

1.2. Wind Engineering Modeling and Analysis Summary ............................................................... 7

1.3. Weathering Steel Modeling and Analysis Summary ............................................................... 7

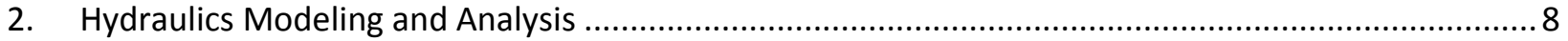

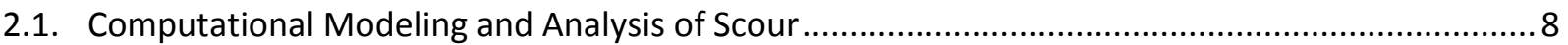

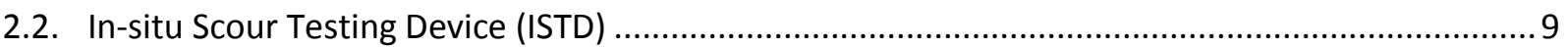

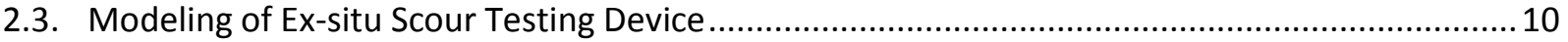

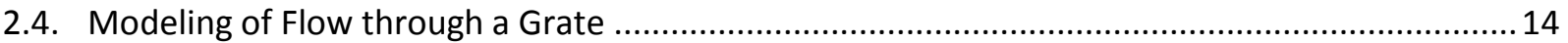

2.5. Options for Parallelization and Running 2D Hydraulics Analysis Software on TRACC Clusters ..... 16

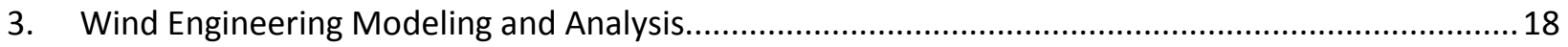

3.1. Test of Coupling STAR-CCM+ with LS-DYNA by Comparing with a Benchmark STAR-CCM+/Abaqus

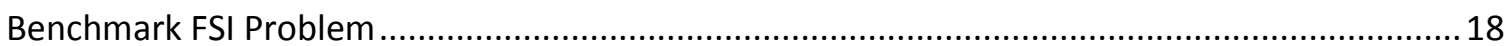

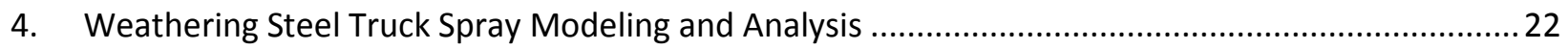

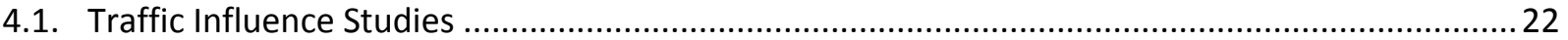

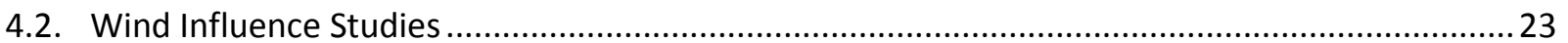

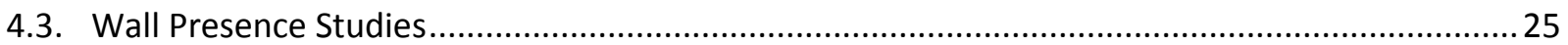

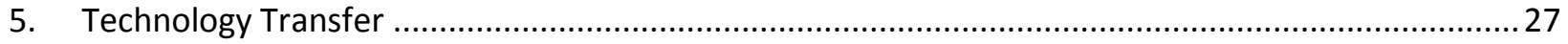

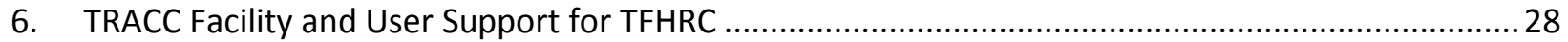

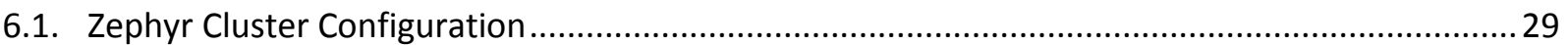




\section{List of Figures}

Figure 2.1 Grainy pattern of particles settling onto the bed of a rectangular channel using the Lagrangian particle transport model 9

Figure 2.2 Diagram of ex-situ scour testing device at the Turner-Fairbank hydraulics laboratory ............10

Figure 2.3 ESTD test section with moving belt in fluid tank ............................................................ 11

Figure 2.4: Geometry used in the 3D modeling of the ESTD ............................................................ 12

Figure 2.5: Velocity distribution plot (top) at time instance of speeding up the belt from $1 \mathrm{~m} / \mathrm{s}$ to $3 \mathrm{~m} / \mathrm{s}$ (bottom) at the end of simulation

Figure 2.6: Wall shear stress on the bottom surface (top) at time instance of speeding up the belt from 1

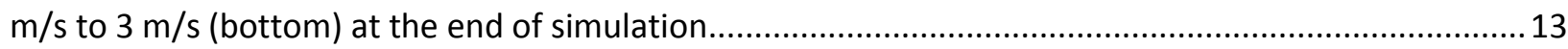

Figure 2.7 Drawing of a grate designed to meet ADA requirements....................................................... 14

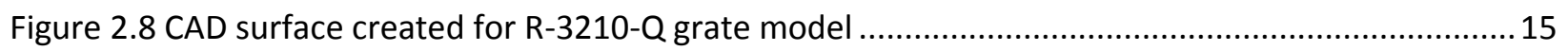

Figure 2.9 Initial simulation showing drainage of a stagnant pool of water above the grate using the multiphase VOF flow model to test this capability for flow into a catch basin with air in most of the upper basin

Figure 2.10 Velocity distribution in slice through grate holes showing jets produced when there is only a downward gravity driven flow..... 16

Figure 3.1 CFD domain and grid for analysis of flexible plate protruding into the flow setup for analysis coupling with LS-DYNA.

Figure 3.2: Morphed mesh at maximum plate deflection in (a) ABAQUS coupling (b) LS-DYNA coupling 19

Figure 3.3: Time history of the plate deflection in LS-DYNA - STAR-CCM+ coupling.....

Figure 3.4: Velocity vector plot in the middle plane at maximum plate deflection ...............................20

Figure 3.5: Pressure distribution in the middle plane at maximum plate deflection

Figure 4.1: Cumulative plot presenting number of parcels at the bridge beam level in basic cases for simulations with one and two trucks.

Figure 4.2: Direction of the wind analyzed for the case with sloped embankment.....

Figure 4.3: Cumulative plot presenting number of parcels at the bridge beam level for simulations with one truck and different wind conditions 
Figure 4.4: Location of the wall for tunneling effect studies....

Figure 4.5: Cumulative plot presenting number of parcels at the bridge beam level for simulations with one truck and different geometry setup underneath the bridge.

Figure 4.6: Parcel count at the bridge beam level in the models with different short vertical wall distance

Figure 5.1 TRACC booth at the 2012 National Hydraulic Engineering Conference ......

Figure 6.1 TRACC Phoenix cluster (left) and Zephyr cluster (right) in the machine room at Argonne's high performance computing facility....

Figure 6.2 Zephyr backplane showing dense cabling due to the dense configuration of 4 compute nodes per $2 \mathrm{U}$ rack in the cabinets.

Figure 6.3 High density of cables at the $40 \mathrm{~GB} / \mathrm{s}$ Infiniband interconnect switches between cluster nodes

Figure 6.4 The Zephyr cluster is powered from two independent circuits. An automatic failover switch provides power to cluster components with a single power supply if one of the supply circuits is interrupted. 


\section{Introduction and Objectives}

The computational fluid dynamics (CFD) and computational structural mechanics (CSM) focus areas at Argonne's Transportation Research and Analysis Computing Center (TRACC) initiated a project to support and compliment the experimental programs at the Turner-Fairbank Highway Research Center (TFHRC) with high performance computing based analysis capabilities in August 2010. The project was established with a new interagency agreement between the Department of Energy and the Department of Transportation to provide collaborative research, development, and benchmarking of advanced three-dimensional computational mechanics analysis methods to the aerodynamics and hydraulics laboratories at TFHRC for a period of five years, beginning in October 2010. The analysis methods employ well benchmarked and supported commercial computational mechanics software. Computational mechanics encompasses the areas of Computational Fluid Dynamics (CFD), Computational Wind Engineering (CWE), Computational Structural Mechanics (CSM), and Computational Multiphysics Mechanics (CMM) applied in Fluid-Structure Interaction (FSI) problems.

The major areas of focus of the project are wind and water effects on bridges - superstructure, deck, cables, and substructure (including soil), primarily during storms and flood events - and the risks that these loads pose to structural failure. For flood events at bridges, another major focus of the work is assessment of the risk to bridges caused by scour of stream and riverbed material away from the foundations of a bridge. Other areas of current research include modeling of the salt spray transport into bridge girders to address suitability of using weathering steel in bridges, CFD analysis of the operation of the wind tunnel in the TFHRC wind engineering laboratory, and coupling of CFD and CSM software to solve fluid structure interaction problems, primarily analysis of bridge cables in wind.

This quarterly report documents technical progress on the project tasks for the period of July through September 2012.

\subsection{Hydraulics Modeling and Analysis Summary}

The primary Computational Fluid Dynamics (CFD) activities during the quarter concentrated on the development of models and methods supporting experimental work at TFHRC and investigating modeling options for adding sediment transport to the mesh morphing scour model. Models of both a 
laboratory device and a field device for measuring the resistance of a sediment material to scour by shear forces have been developed in order to test operating procedures and design options. An investigation of option to use either the Eulerian or Lagrangian multiphase particle transport models was carried out. While the physics of both models are well developed and present in the STAR-CCM+ software, methods to move particles in and out of the system undergoing scour through a rough wall constituting the sediment bed where scour and settling back of sediment particles is taking place are not obvious or straightforward to implement. Efforts to resolve the issues are ongoing. A presentation of most of the major CFD modeling projects carried out at TRACC in support of the research and development program at TFHRC was made at the 2012 National Hydraulics Conference in August, and TRACC CFD staff was present with the TRACC booth. Both appreciation of the capabilities and benefits of CFD analysis applied to hydraulics problems and the use of CFD analysis have increased significantly since the last conference in 2010.

\subsection{Wind Engineering Modeling and Analysis Summary}

The current work supporting wind engineering modeling and analysis efforts is focusing on validating the coupling procedure developed by TRACC staff to tie together the CFD and CSM codes (STAR-CCM+ and LS-DYNA) for analyzing various Fluid Structure Interaction (FSI) problems. A benchmark problem was selected from the STAR-CCM+ manual and the analysis attesting validity of the coupling procedure was performed.

\subsection{Weathering Steel Modeling and Analysis Summary}

In the current quarter the simulation work on weathering steel truck spray modeling was finalized. TRACC staff presented results of that work at the Bridge Engineering Task Force Meeting that took place on August 9th, 2012, in Chicago. The current report highlights the results focusing on explaining the influence of the traffic, wind, and different geometries of approach to the bridge on the amount of salt spray particles transported to the bridge beams. 


\section{Hydraulics Modeling and Analysis}

\subsection{Computational Modeling and Analysis of Scour}

Three-dimensional scour modeling at a cylindrical pier using the mesh morphing capabilities in STAR$\mathrm{CCM}+$, including a primitive sand slide model have been successful in coming close to experimental results in the bowl shape of the scour hole. The depth of the scour hole has been over predicted and downstream pile up of eroded sediment is absent, because the model does not include multi-phase sediment transport. STAR-CCM+ includes well developed multiphase particle transport models including Eulerian and Lagrangian approaches, where the Eulerian approach treats particles as a dispersed continuum and solved partial differential equations for the particle concentration, and momentum of particles in a computational cell, while the Lagrangian approach tracks particles through the system, solving the ordinary differential equations of Newton's laws of motion.

Approaches to adding sediment transport to the scour model are currently being investigated. Both Eulerian and Lagrangian approaches are being considered and there are difficulties with either approach in applying them to modeling scour.

Using Eulerian multiphase particle transport appears to be a good option because the particle flux (in terms of number density) is a continuous and consequently a reasonably smooth function. The problem encountered in trying to use the Eulerian multiphase model was how to have a particle flux coming from a wall boundary. A volume source term could be created for particles, but there is not a clear way in the documentation to compute this source term as a field function of the bed shear stress only in computational cells that have a cell face that is part of the eroding river or flume bed. Ways to do this are still being explored in consultation with CD-adapco technical support.

The other option under investigation is to use Lagrangian multiphase particle or parcel tracking. It appears that the river bed, a rough wall boundary, can be defined as an injector or set of injectors for particles, and that a flow rate specification can be set to a mass flux, where the mass flux is a field function of the mean shear stress on the injector surface. The problem here is that it is desired to have injection rate vary over the bed cell faces as a function of the local shear stress at the centroid of each bed cell face on the surface subject to scour shear stress. To accomplish this degree of resolution of the particle entrainment rate from an eroding surface appears to require that each cell face be defined as a separate particle injector, which is not feasible when there are thousands and even tens of thousands of computational cell faces on the eroding surface mesh. Another possible problem is that the riverbed erosion rate at a cell face in a time step can be slow - so that only a fraction of a sand particle mass gets entrained in a time step. It appears that a parcel can be injected that represents only a fraction of one particle as opposed to hundreds or thousands of particles, but this capability has not yet been tested. The third problem in using a Lagrangian approach for sediment transport is that a smooth function of the particle settling rate on the riverbed is needed to balance with the erosion rate due to shear. There is a field function, "Incident Mass Flux of Phase 1" that does give mass flux on cell faces on the riverbed, however, it appears to be very grainy in our tests - one cell face might have a large mass flux because 
one or several parcels have hit the face while an immediate neighbor of a high incident flux face may have a zero incident flux. This pattern can be seen in Figure 2.1 and is in part a consequence of the limitations of the number of particles that can be tracked with the Lagrangian model. While the system may contain millions or even billions of particles, computational resources and time constraints limit the number of Lagrangian particles tracked to a range of ten thousand to a couple of hundred thousand, a number that can normally be considered statistically significant in determining the particle transport processes in a system. The problem in this case is that some method of smoothing probably needs to be applied to the settling rate at the surface to obtain a physically realistic distribution of the settling rate on the surface that will yield a numerically stable combined entrainment and settling rate to be used to determine bed displacements over a time step. This issue will have to be resolved if the Lagrangian approach is used for sediment transport.

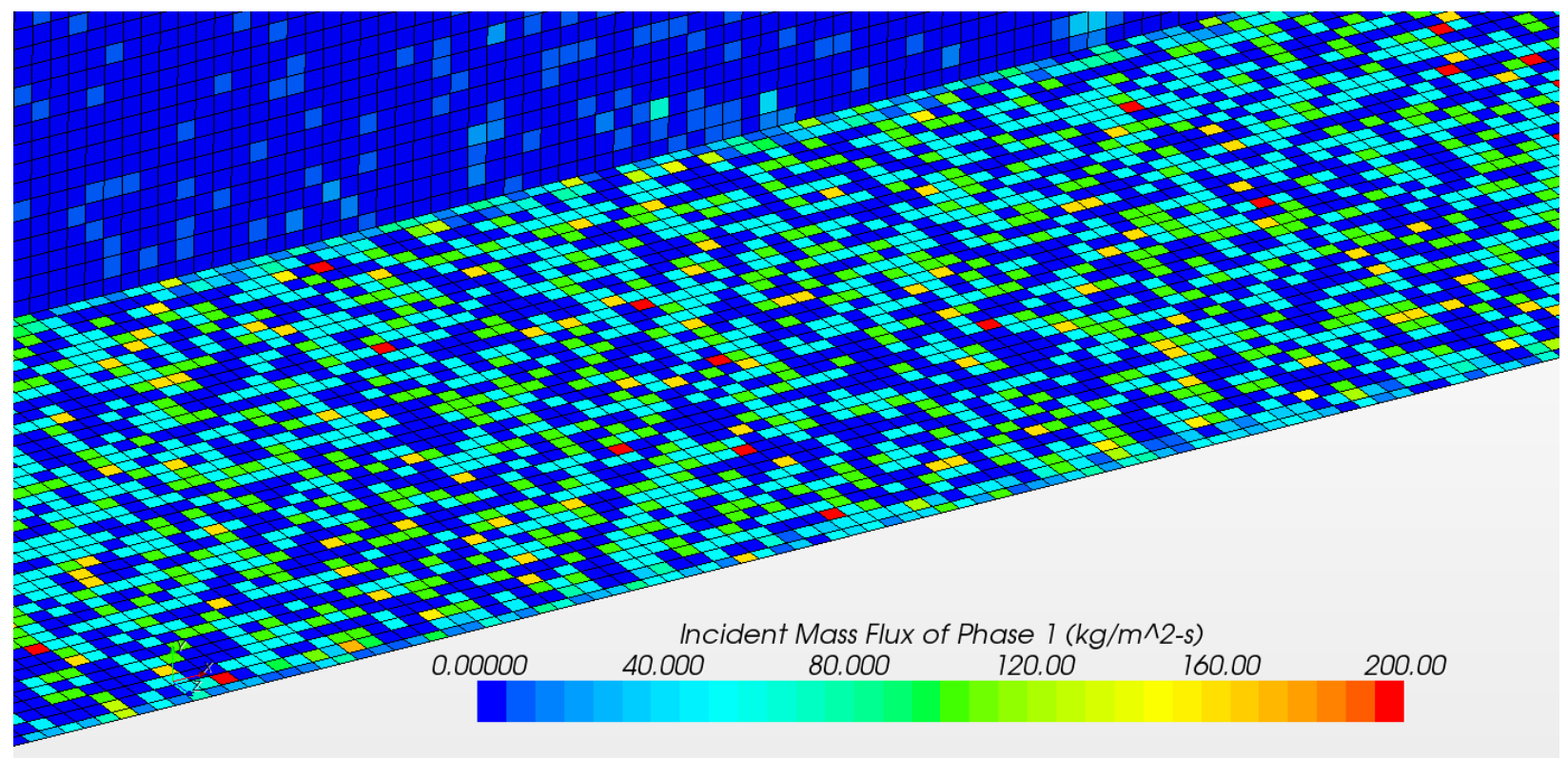

Figure 2.1 Grainy pattern of particles settling onto the bed of a rectangular channel using the Lagrangian particle transport model

\subsection{In-situ Scour Testing Device (ISTD)}

Model development and evaluation continued for various alternatives for an in-situ scour testing device being designed at TFHRC to test erosion rates of sediments in riverbeds in the field. The design and models have evolved through several very different ideas until one that is both simple and practical was developed. TFHRC is in the process of patenting the final version of the device. For this reason, details of the modeling effort and results will be deferred and reported after patent documents on the device have been filed. 


\subsection{Modeling of Ex-situ Scour Testing Device}

An ex-situ scour testing device at TFHRC was designed to produce high shear with a turbulent velocity profile in a confined space by super imposing coquette flow with pressure driven flow through a rectangular channel. A diagram of the device is shown in Figure 2.2. An expanded diagram of the test section with moving belt is shown in Figure 2.3

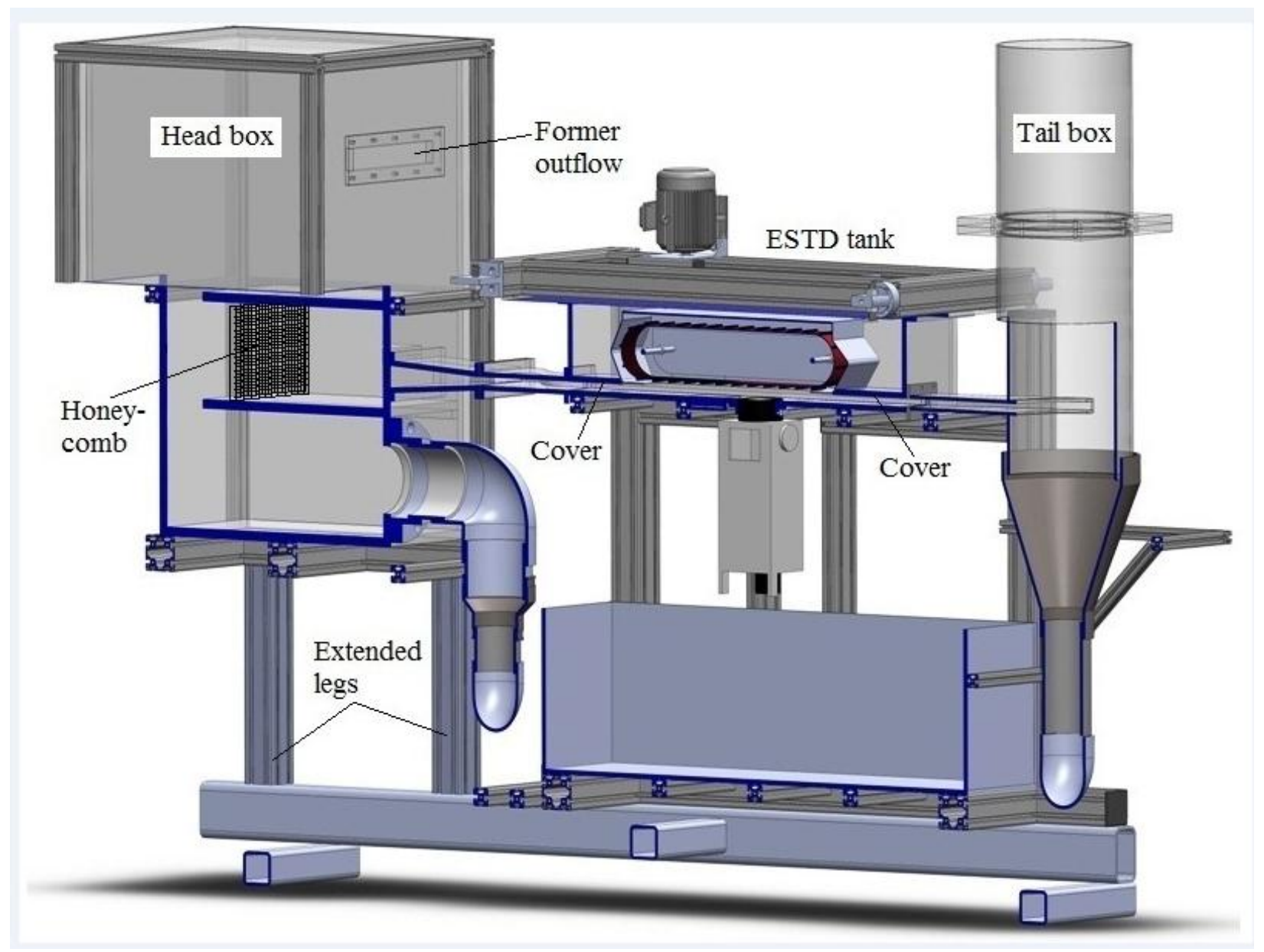

Figure 2.2 Diagram of ex-situ scour testing device at the Turner-Fairbank hydraulics laboratory 


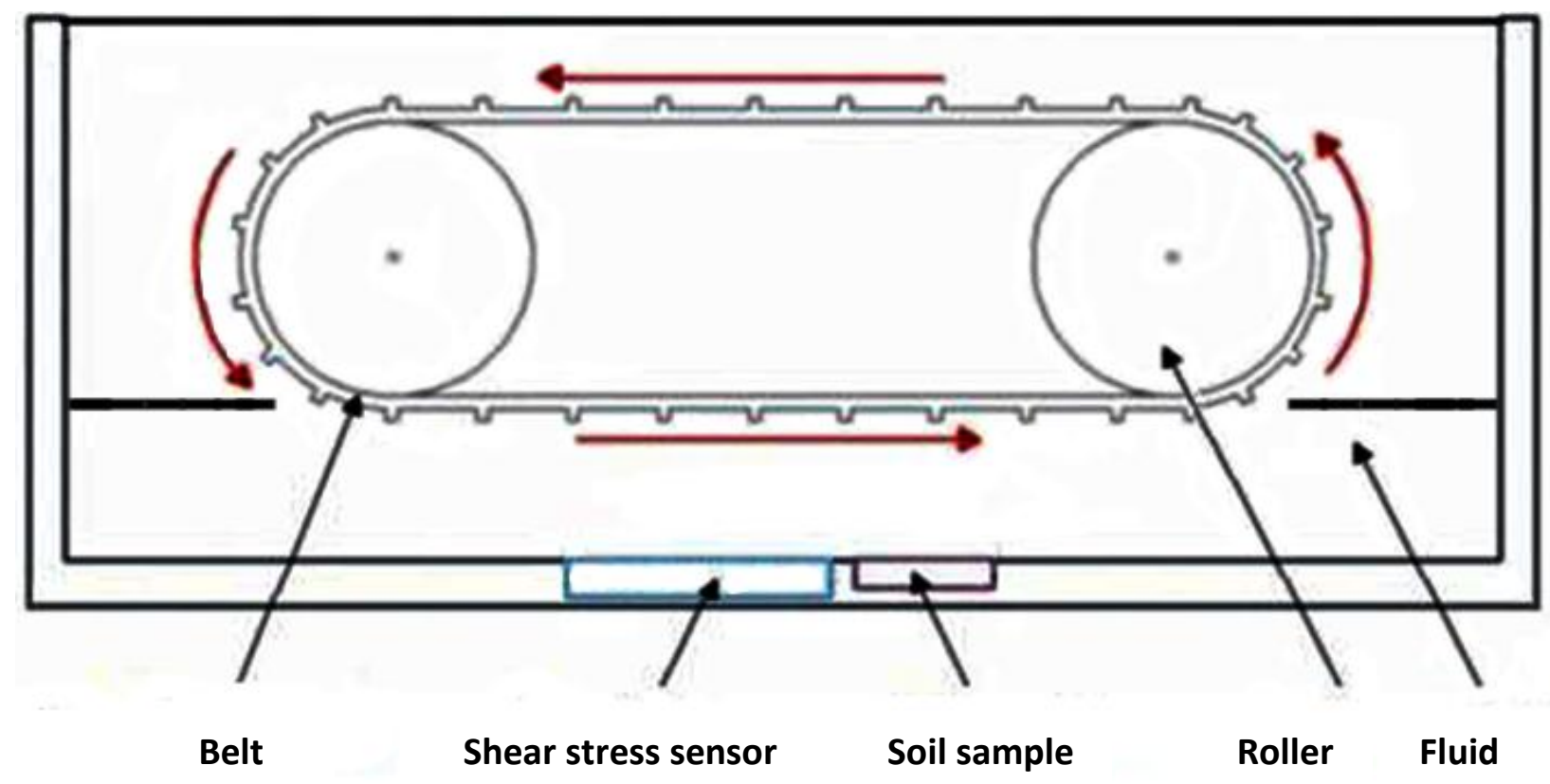

Figure 2.3 ESTD test section with moving belt in fluid tank

The rectangular channel length is about $1 \mathrm{~m}$. The channel has a height of $2 \mathrm{~cm}$ and a width of $12 \mathrm{~cm}$. The belt width is $10 \mathrm{~cm}$, and the test section opening that the belt traverses is $46.2 \mathrm{~cm}$ long. The rubber ribs are $5.1 \mathrm{~mm}$ high, and the distance between ribs is $4 \mathrm{~cm}$. The test section contains a sediment soil sample tray and shear stress sensor immediately upstream of the sample tray.

A CFD model of the device is being built to test the effects of various combinations of pressure flow through the channel and belt speeds on the shear stress and velocity profile at the soil sample tray. The modeling task is challenging as a consequence of the moving belt boundary. The STAR-CCM+ software does have a variety of motion modeling capabilities, however, moving the belt and rollers is a combination of rotational and linear motion that is not possible to set up in a straight forward way. The initial modeling effort, described here, uses the sliding mesh capability to create a moving boundary surface with ribs that moves through the test portion of the domain. To accomplish this, the domain is built with two regions as shown in Figure 2.4. The upper region is a very long channel with the ribs of the belt and the cavities between the ribs. The lower region is the test section channel. The two regions are connected with an in place interface that extends the length $(46.2 \mathrm{~cm})$ of the opening between roller and belt domain and the rectangular flow channel. The yellow dashed lines in Figure 2.4 show the zone where the two regions are open to interaction. In the sliding region, the rib and belt boundaries are smooth walls and all other boundaries are symmetry planes, except for the zone where the lower boundary shares an in place open interface with the channel. For the channel region, the inlet boundary condition is a specified uniform velocity and the outflow boundary is a pressure boundary. The sides are treated as symmetry planes because the full channel width was not modeled; only a narrow width section at the center of the channel was included in this initial model. 
- A 3D model has been built with a sliding mesh subdomain to model motion of the ribbed belt. The initial condition for velocity is $2.5 \mathrm{~m} / \mathrm{s}$.

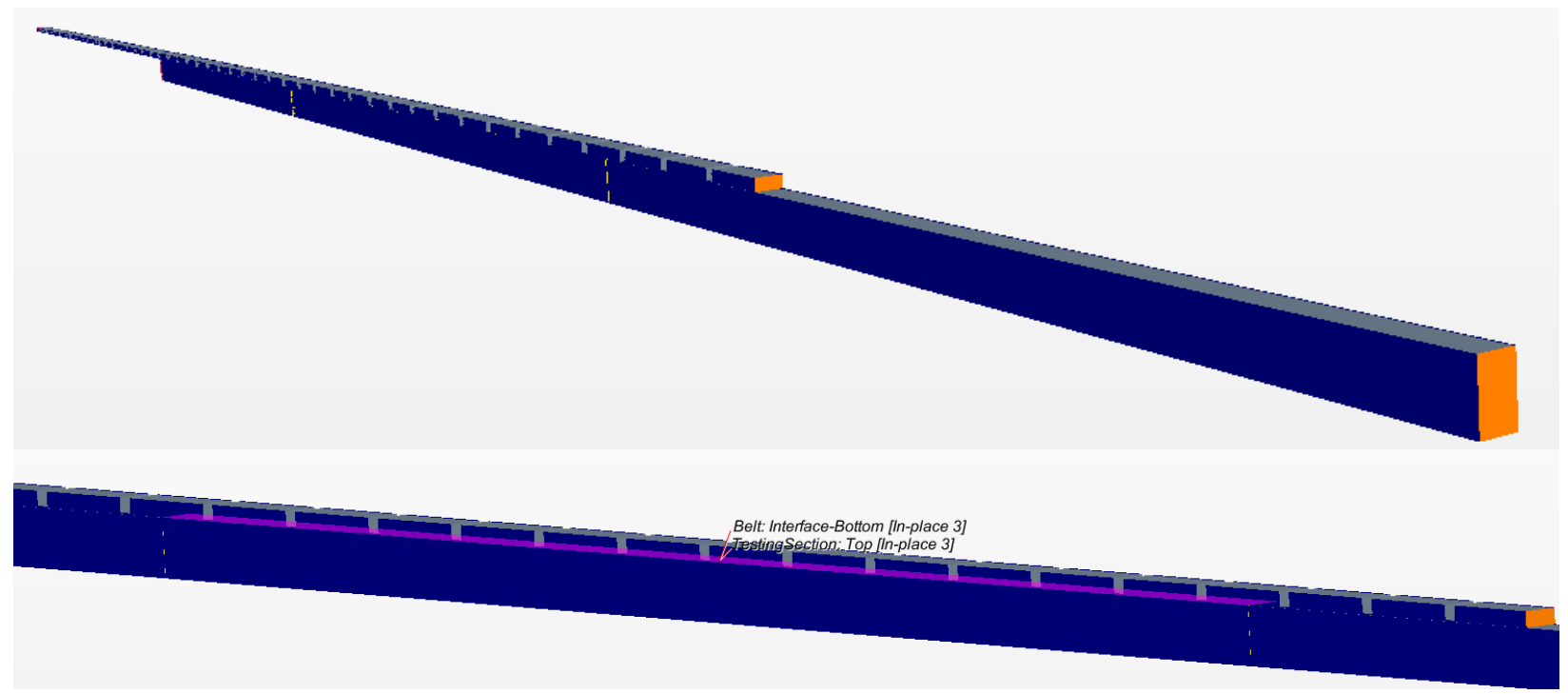

Figure 2.4: Geometry used in the 3D modeling of the ESTD

Due to the motion of the ribbed region, the analysis is unsteady. The results shown here are for a case with an inlet flow channel velocity of $2.5 \mathrm{~m} / \mathrm{s}$ that was restarted from a case that had the belt moving at a constant $1 \mathrm{~m} / \mathrm{s}$. At time zero, the ribbed sliding mesh velocity is changed from $1 \mathrm{~m} / \mathrm{s}$ to $3 \mathrm{~m} / \mathrm{s}$. The top and bottom color plots of velocity in Figure 2.5 cover the test section. The top one is just after the ribbed belt velocity is changed from $1 \mathrm{~m} / \mathrm{s}$ to $3 \mathrm{~m} / \mathrm{s}$ showing a disturbance in the velocity field caused by the change in belt velocity. The bottom plot shows the velocity field under the moving ribs after a long period of time. It changes only slowly in the flow direction and does not show a strong variation under a rib as compared to the lower region under a cavity between ribs.

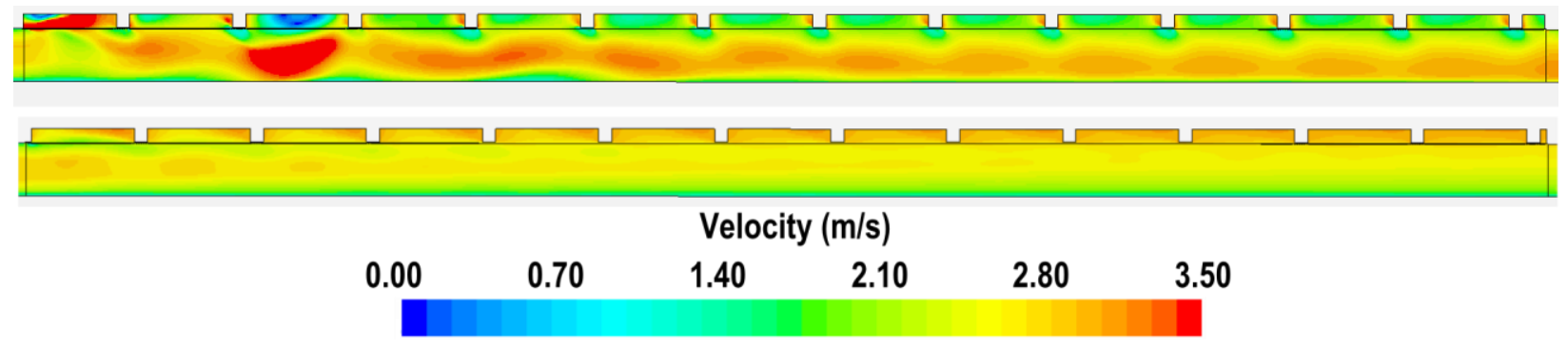

Figure 2.5: Velocity distribution plot (top) at time instance of speeding up the belt from $1 \mathrm{~m} / \mathrm{s}$ to $3 \mathrm{~m} / \mathrm{s}$ (bottom) at the end of simulation 
The shear stress along the test section on the bottom of the channel is shown in the upper plot of Figure 2.6 just after the ribbed belt velocity change and the lower plot is shown at a time when the transient introduced by the change in belt velocity to $3 \mathrm{~m} / \mathrm{s}$ has decayed. At that time the shear stress on the channel bottom is nearly uniform over the position of the test sample tray and does not exhibit any significant periodic oscillation due to the moving ribs.
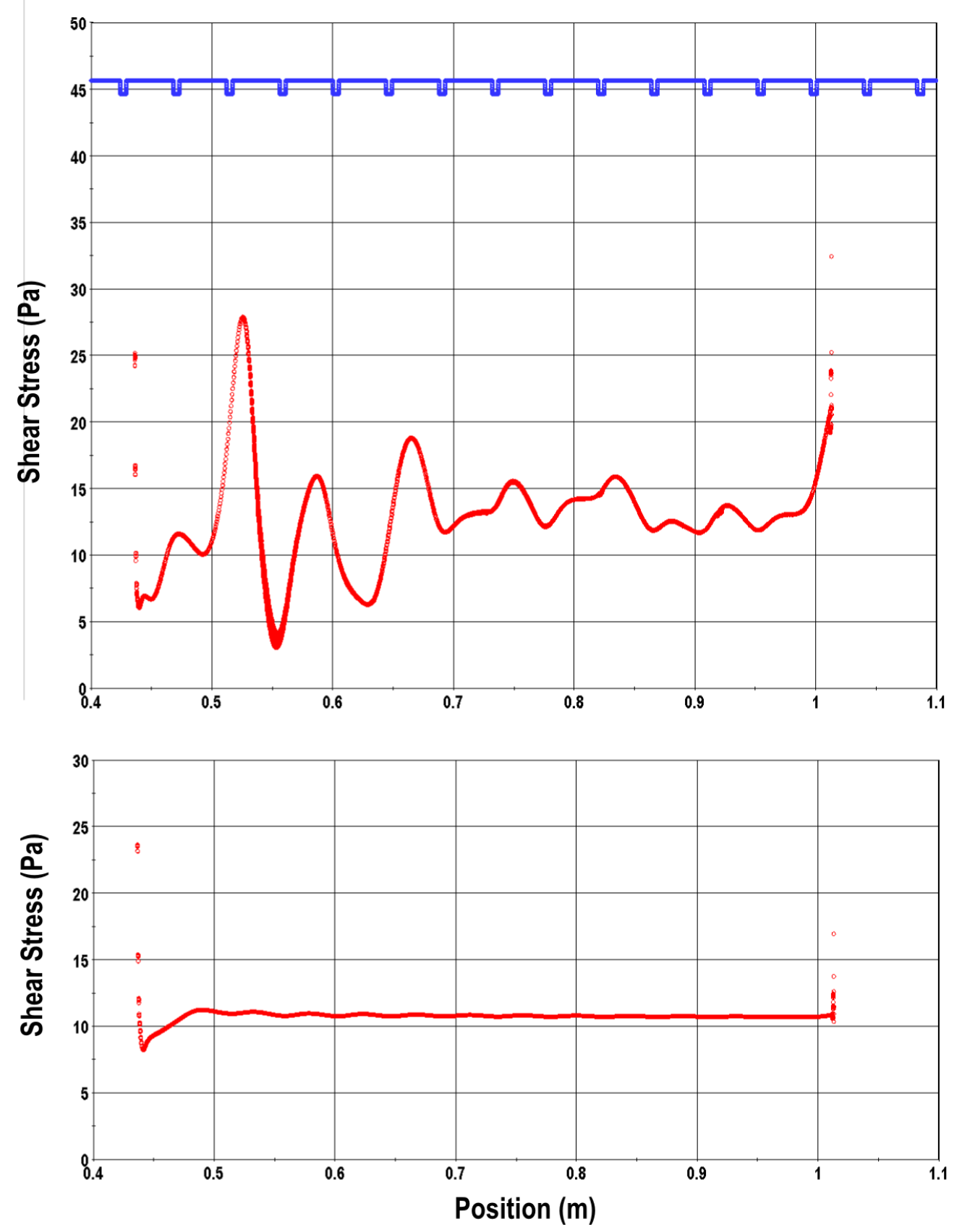

Figure 2.6: Wall shear stress on the bottom surface (top) at time instance of speeding up the belt from $1 \mathrm{~m} / \mathrm{s}$ to $3 \mathrm{~m} / \mathrm{s}$ (bottom) at the end of simulation 


\subsection{Modeling of Flow through a Grate}

An initial modeling effort was begun to demonstrate CFD analysis capabilities for flow through a storm drain grate in the street. Newer storm drain grate designs contain smaller slots to meet the standards set in the Americans with Disabilities Act (ADA) and to improve the safety of grates in streets in general. For example, not only wheelchair wheels but also large numbers of bicycles have thin wheels that may drop several inches into the slots in older drain grates posing a hazard for people riding bicycles near the side of the road. An alternative to testing designs with smaller slots in laboratory flumes is to build a full scale computational model that has a geometry and flow rates that match the conditions that would be present in a street installation of a storm drain grate and then use CFD analysis to determine the fraction of flow along the street entering the catch basin through the grate and other parameters of interest. Initial testing was done to verify that the multiphase VOF model would perform adequately in this type of flow system. VOF models are typically used for free surface flows and solve only one momentum equation even though both air and water are in the system because the small computational cells typically contain only water or air. In cells that are cut by the free surface, the air and water fluid properties are typically averaged to obtain fluid properties used in the solution of the momentum equation. VOF models would typically not be used to model sprays that result from jet breakup. Flow through a grate with slots will generate a set of slot jets that are likely to breakup somewhat as water flows into the catch basin. This breakup may not be handled accurately by a VOF model, however, it occurs in the catch basin downstream of the region of interest and is not likely to affect the computation of the amount of water entering the grate. The initial test documented here was intended to verify that using a VOF model for analysis of flow into a grate is numerically stable and yields qualitatively realistic results. A grate for this test was taken from [1] and its geometry is shown in Figure 2.7.
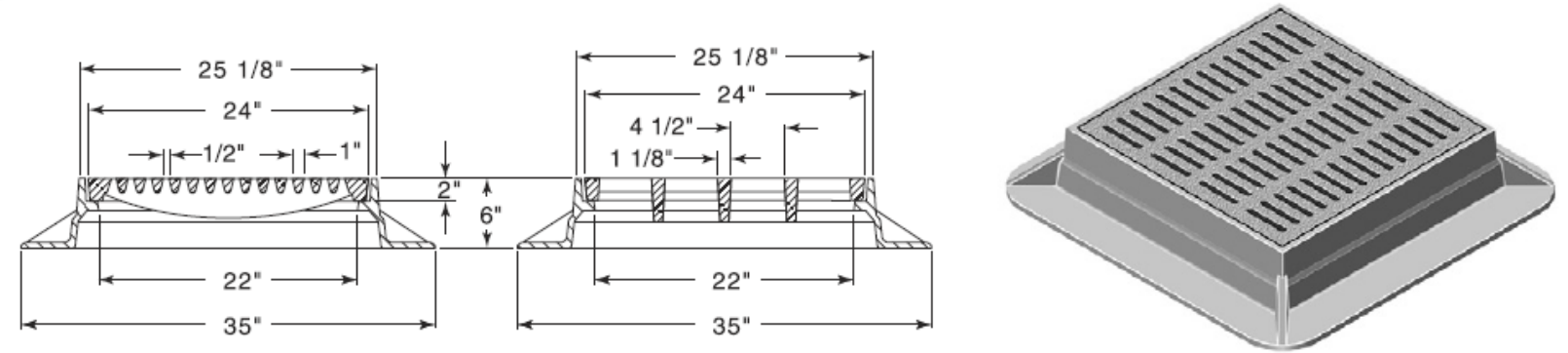

Figure 2.7 Drawing of a grate designed to meet ADA requirements

The surface geometry created for the initial testing is shown in Figure 2.8. The first tests are simply for gravity driven flow from a pool of water above the grate into a catch basin containing air below the grate. This type of flow will create a set of slot jets of water falling into the catch basin. Both the top inlet boundary and the bottom outflow boundary are pressure boundaries. The side boundaries were walls. 


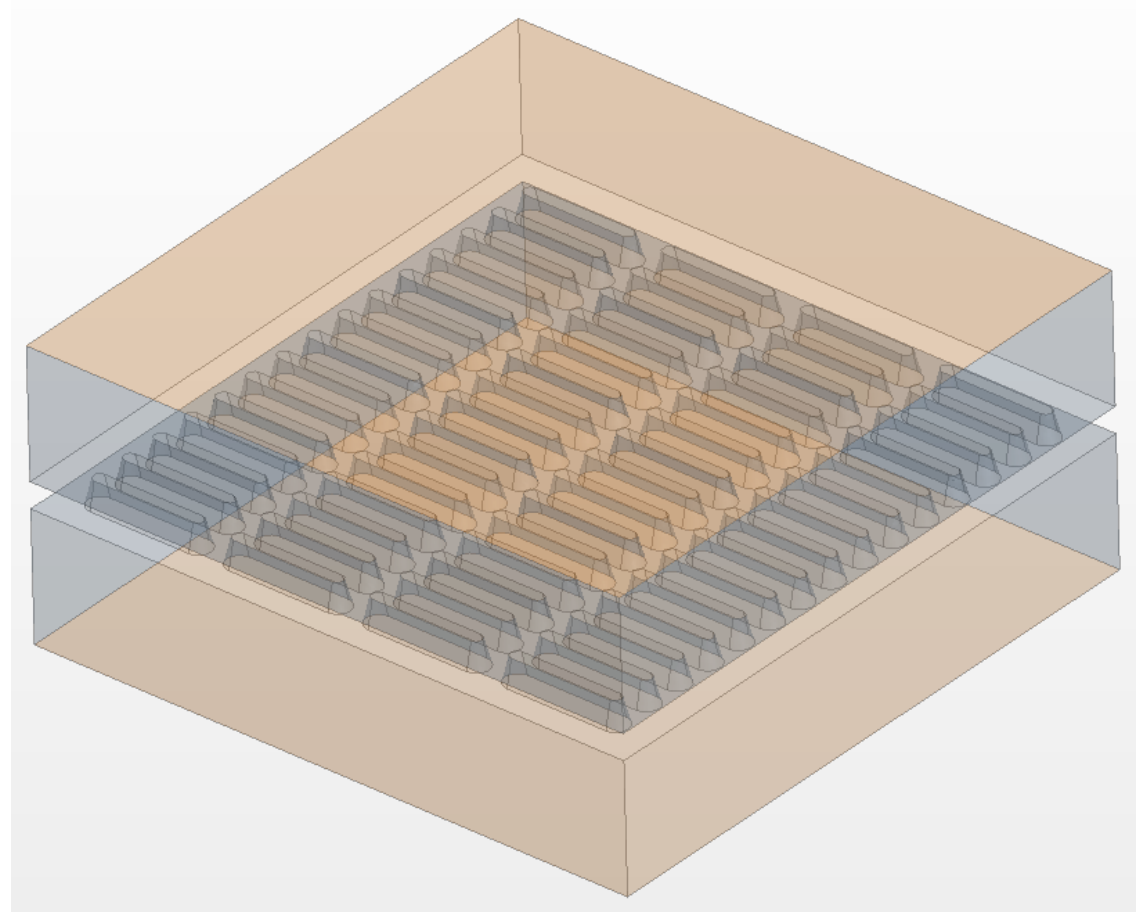

Figure 2.8 CAD surface created for R-3210-Q grate model

Results are shown in Figure 2.9 and Figure 2.10 with color plots of the velocity of the slot jets. Figure 2.9 shows a color plot of velocity on the iso-surfaces approximately separating air and water below the grate. Also included is a velocity plot on a plane slice through a row of grate slots. This plot is shown in Figure 2.10 as a 2D plot.

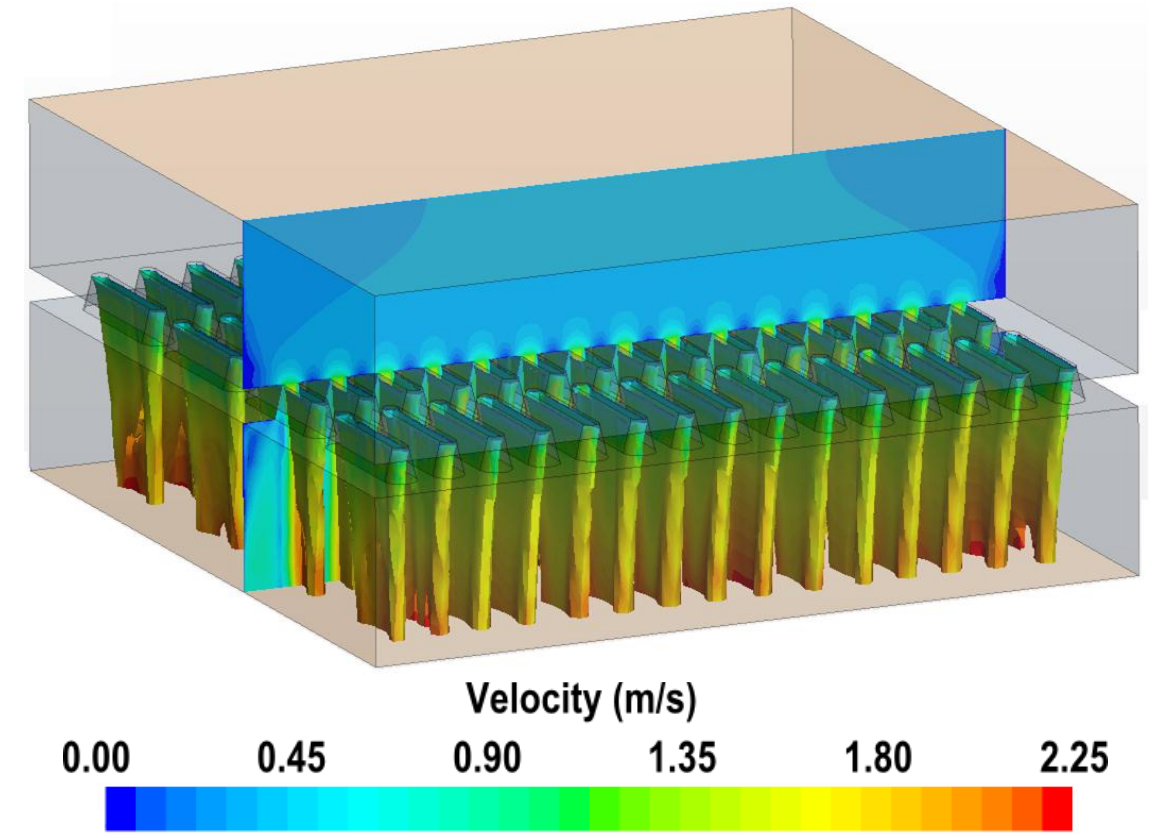

Figure 2.9 Initial simulation showing drainage of a stagnant pool of water above the grate using the multiphase VOF flow model to test this capability for flow into a catch basin with air in most of the upper basin 


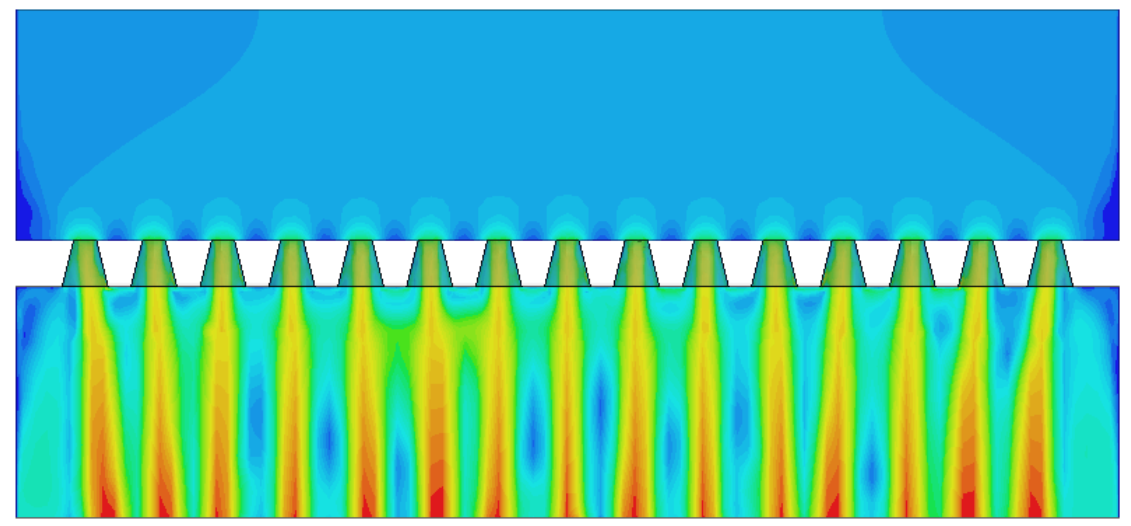

\section{Velocity $(\mathrm{m} / \mathrm{s})$}

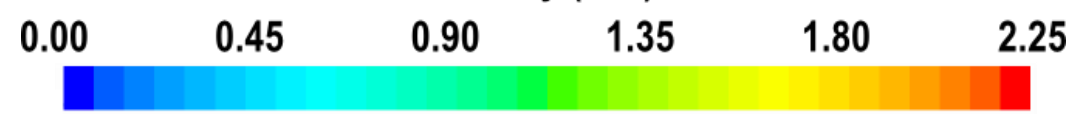

Figure 2.10 Velocity distribution in slice through grate holes showing jets produced when there is only a downward gravity driven flow

\subsection{Options for Parallelization and Running 2D Hydraulics Analysis Software on TRACC Clusters}

A brief initial investigation of options for making one or more 2D hydraulics analysis software solver packages available on the TRACC clusters to provide access for field engineers to high performance computing facilities for the solution of large 2D hydraulics problems was done. There are two primary approaches to implementing parallelism in a serial program that runs on only one processor and one core of a multi-core processor. These approaches are using the industry standard message passing interface (MPI) to implement parallelism and alternatively using multi-threading to implement parallelism.

The MPI standard was developed before multi-core processors existed, and it was developed to provide parallel processing on systems ranging from one dual processor PC or a few PCs networked together in a small organization to large high performance clusters. MPI works well on both shared memory systems and distributed memory systems. In a shared memory system, each process (core) simply has access only to the memory assigned to it. Preventing race conditions and deadlocks is not a significant issue with MPI because each process (core) only has access to its own assigned memory space on either shared or distributed memory systems. When processes do need to obtain variable values from other processes, for example the values of spatial or logical neighbors at a subdomain boundary that reside in the memory space of another process, these variable values are exchanged via the message passing paradigm using MPI. Thus instead of having critical code segments that must be locked allowing only one thread to execute the segment at a time to avoid race conditions that could corrupt shared memory that is read and written by more than one process, which can be bottlenecks with their own inefficiencies, when using MPI, the small amount of memory with values that need to be shared among processes is duplicated in the memory space of each process and kept up to date with message passing. 
The major CFD companies use MPI for their solvers, and for the cases where small problems are solved on single dual core PCs, the speed up in using both cores is nearly a factor of 2 , which is excellent.

The development of hybrid algorithms that use both MPI with domain decomposition in what is sometimes referred to as coarse grained parallelism and multi-threading using OpenMP, pthreads, etc. for what may be termed fine grain parallelism is an area of active research that is still complex and challenging to get right. The major commercial CFD companies are still nearly universally using an MPIeverywhere approach because it works very well on everything from single dual or quad core PCs to large clusters.

Either of the two primary approaches applied to a 2D serial, depth averaged, hydraulics flow code would likely yield software that would run well on either of the TRACC clusters. A multi-threaded code that does not use MPI would be restricted to running on a single compute node on the TRACC clusters. The Phoenix cluster would be able to handle a problem up to about 5 million grid points on an 8 GB RAM single compute node for either an MPI code or a multi-threaded code. The Phoenix cluster also has 232 GB RAM nodes that could be used for problems up to about 30 million grid points. The Zephyr cluster's standard compute node has $32 \mathrm{~GB}$ of RAM and 16 floating point cores on 2 processors. Thus, 2D hydraulics problems could be solved that contained up to about 30 million grid points. The Zephyr cluster also has four larger memory nodes, two with 64 GB or RAM and two with 128 GB of RAM. Thus 2D problems up to about 60 million and 120 million grid points could be handled on these nodes respectively. These are only order of magnitude estimates based on 3D analysis memory usage. The estimates should therefore be conservative. Note that it cannot be assumed that 2D problems require $2 / 3$ the memory of 3D problems, because the depth of flow at each point is tracked in a 2D problem, and there may be other memory consuming variables that are carried in a $2 \mathrm{D}$ computation that are not carried explicitly in a 3D problem. Even with the uncertainty of these estimates, it is clear that very large 2D problems can be solved on the TRACC Zephyr cluster using any of the 32 GB nodes, or one of the larger nodes for extremely large problems. The time to solve the problem has not been addressed here. Compared to running a serial 2D depth averaged code, however, using either MPI or a fully multithreaded approach should yield at least an order of magnitude speed up when running on a Zephyr compute node with 16 FPU cores.

The limitation of the multi-threaded approach is that it limits the software to run on a single shared memory computer, either one TRACC cluster compute node or one office desktop machine. As noted above most of the Zephyr compute node far surpass the compute power and memory available on typical office desktop computers, even high end workstations. A major advantage of using MPI in implementing parallelism is that the limitation to run on a single shared memory computer is removed. A 2D depth averaged hydraulics code that uses MPI for parallel computation, could easily be run on 4 quad-core office workstations with $8 \mathrm{~GB}$ of RAM each tied together with $1 \mathrm{~GB} / \mathrm{s}$ Ethernet to solve a problem that would otherwise require a high performance compute node on TRACC's Zephyr cluster.

[1] Neenah Foundry, http://www.nfco.com/municipal/products/combination-inlets/without-curbbox/r-3210-q 


\section{Wind Engineering Modeling and Analysis}

\subsection{Test of Coupling STAR-CCM+ with LS-DYNA by Comparing with a Benchmark STAR-CCM+/Abaqus Benchmark FSI Problem}

In the previous quarter work on multiphysics modeling of bridge cable vibrations has been started. The main tasks for this initial work was to explore coupling capabilities between STAR-CCM+ and LS-DYNA. After contacting CD-adapco, the developer of STAR-CCM+, it was established that there is a possibility to couple the two codes by using file exchange between them. The coupling scripts based on Python scripting language and java macros in STAR-CCM+ have been developed and initial runs proving the concept were performed. The scripts are automatically transferring pressure calculated on the surface of the structure (cable) to LS-DYNA where they are used as an input for structural analysis. The deformed shape of the cable is then transferred back to STAR-CCM+ where it is used for morphing the previous mesh to the new configuration. Then the flow around the structure is calculated again and the loop is repeated for the requested number of times. Since this is not the standard procedure of coupling, additional testing had to be done in order to prove that the procedure not only executes properly but also gives valid results. For that reason another problem was analyzed that was previously solved for coupling of STAR-CCM+ with ABAQUS code and the results were presented in the STAR-CCM+ manual. The coupling with ABAQUS is done internally - no scripting is required. The coupling in LS-DYNA requires the user to develop coupling scripts. The problem pertains to vibrations of a flexible rectangular plate as a result of $10 \mathrm{~m} / \mathrm{s}$ wind blowing on it. The plate was constrained with its bottom edge perpendicular to the flow direction. The size of the modeled domain was respectively: width $0.29 \mathrm{~m}$, height $0.2 \mathrm{~m}$, length $0.9 \mathrm{~m}$. The plate was: $0.1 \mathrm{~m}$ high, $0.08 \mathrm{~m}$ wide and $0.0025 \mathrm{~m}$ thick. Rubber like material with elastic properties was used to model the plate material with the following material constants: Young's modulus $38.4 \mathrm{MPa}$, Poisson's ratio of 0.3 and density equal to $4096 \mathrm{~kg} / \mathrm{m}^{3}$. The plate was located $0.4 \mathrm{~m}$ from the inlet (see Figure 3.1).

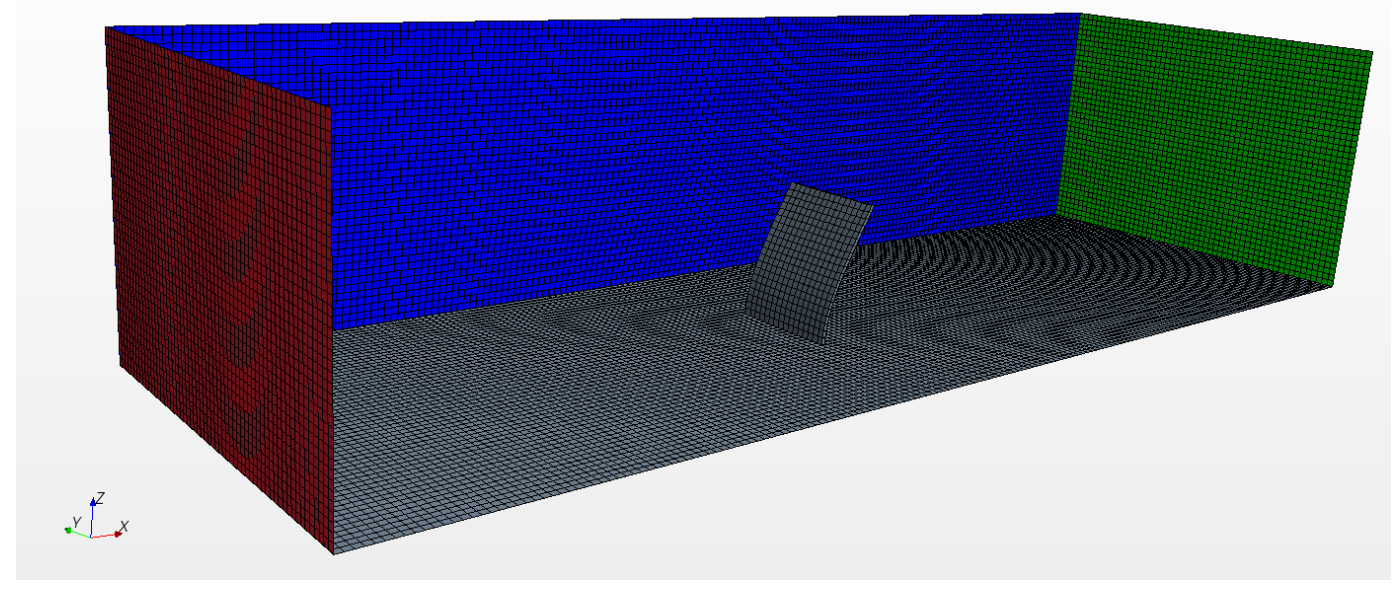

Figure 3.1 CFD domain and grid for analysis of flexible plate protruding into the flow setup for analysis coupling with LS-DYNA 
Figure 3.2 shows the mesh in proximity of the deformed plate for the coupling with ABAQUS and LSDYNA. CD-adapco modeled the domain with polyhedral cells while here it was modeled with hexahedral cells. The overall model was built with around 300,000 cells. The shown configuration is captured at the state of the largest deflection of the plate. Figure 3.3 shows the displacement history of the two top corners of the plate. The maximum displacement was slightly above $0.04 \mathrm{~m}$. With the appropriate damping of oscillations the history of the vibration of the plate was following exactly the results presented by CD-adapco in their example of coupling STAR-CCM+ with ABAQUS software.
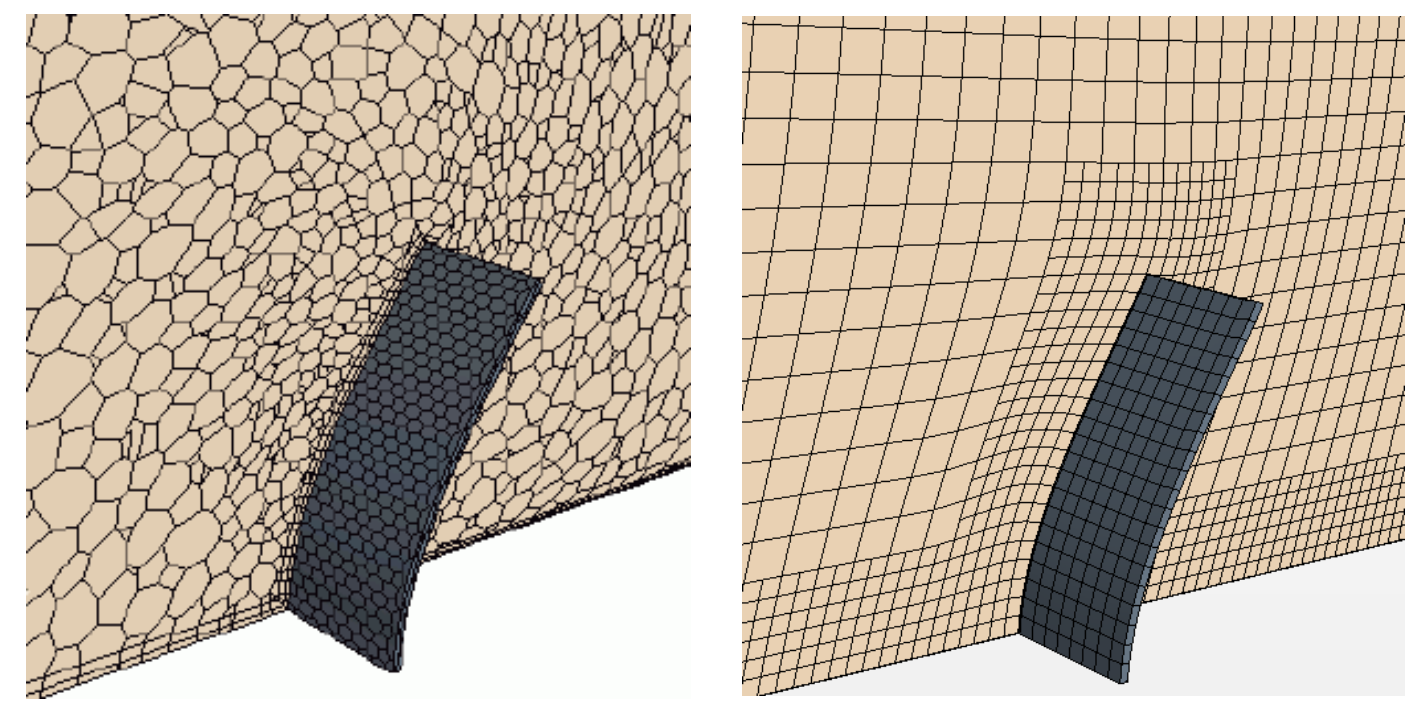

Figure 3.2: Morphed mesh at maximum plate deflection in (a) ABAQUS coupling (b) LS-DYNA coupling

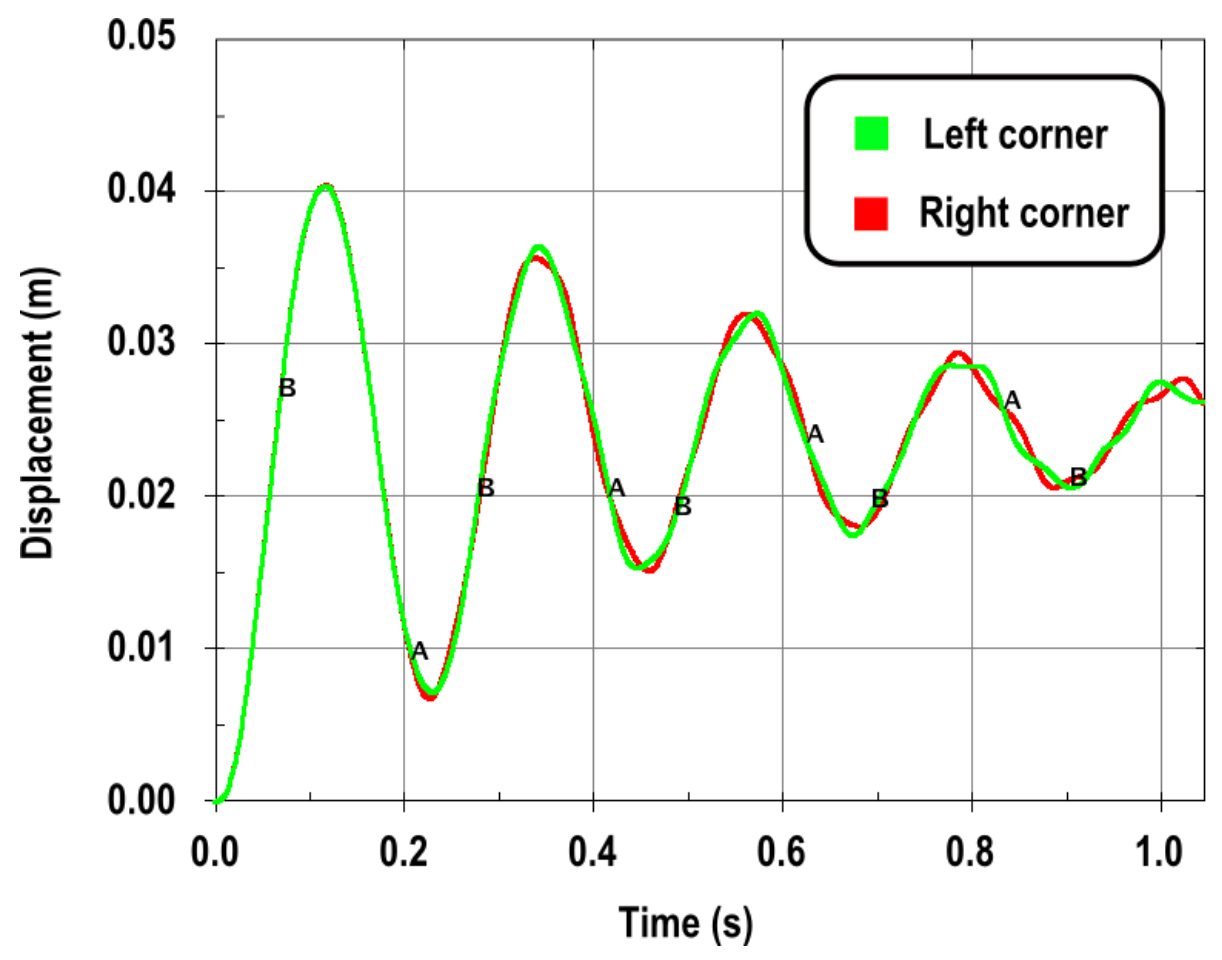

Figure 3.3: Time history of the plate deflection in LS-DYNA - STAR-CCM+ coupling 
Figure 3.4 shows velocity vector plot on the surface cutting the domain in half in the direction of air flow.

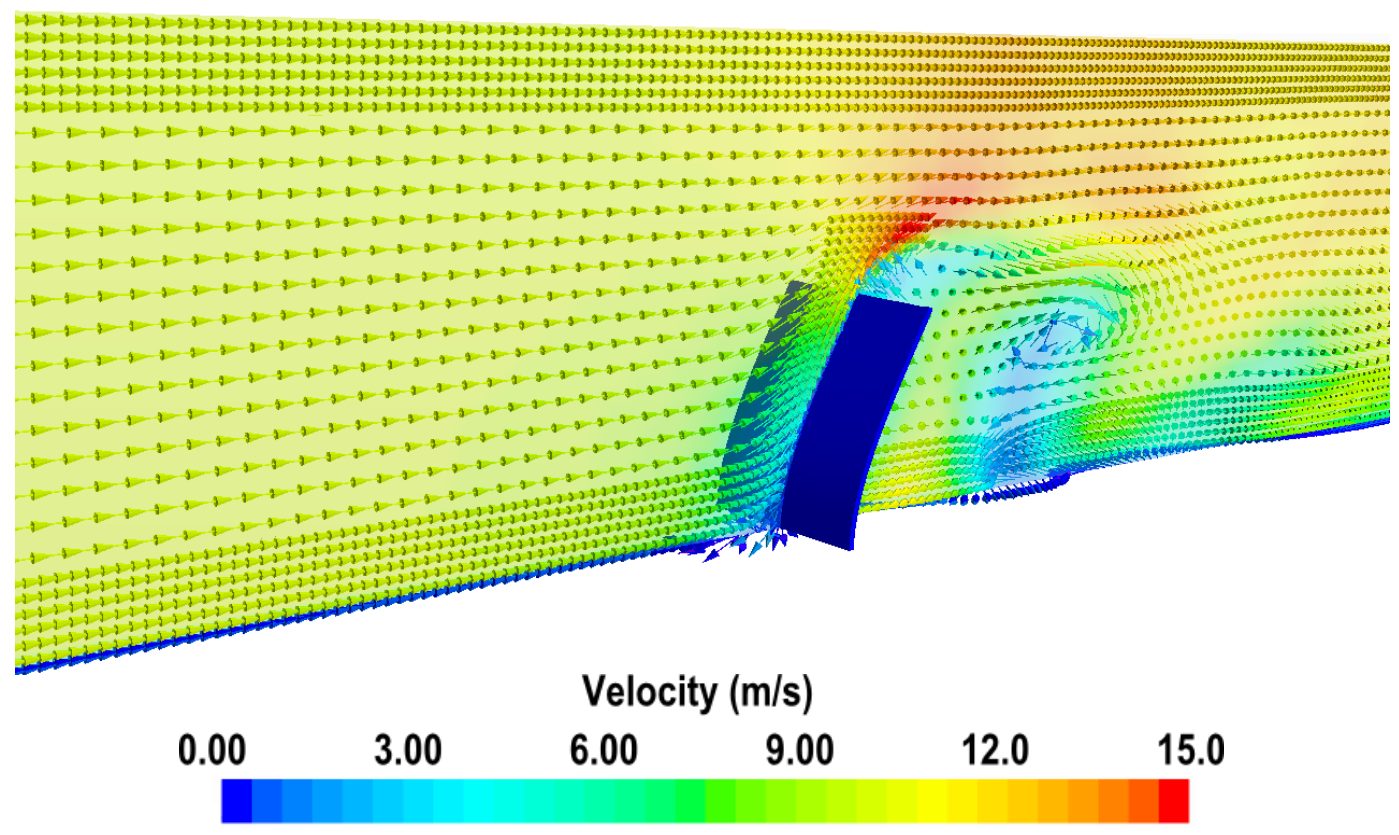

Figure 3.4: Velocity vector plot in the middle plane at maximum plate deflection

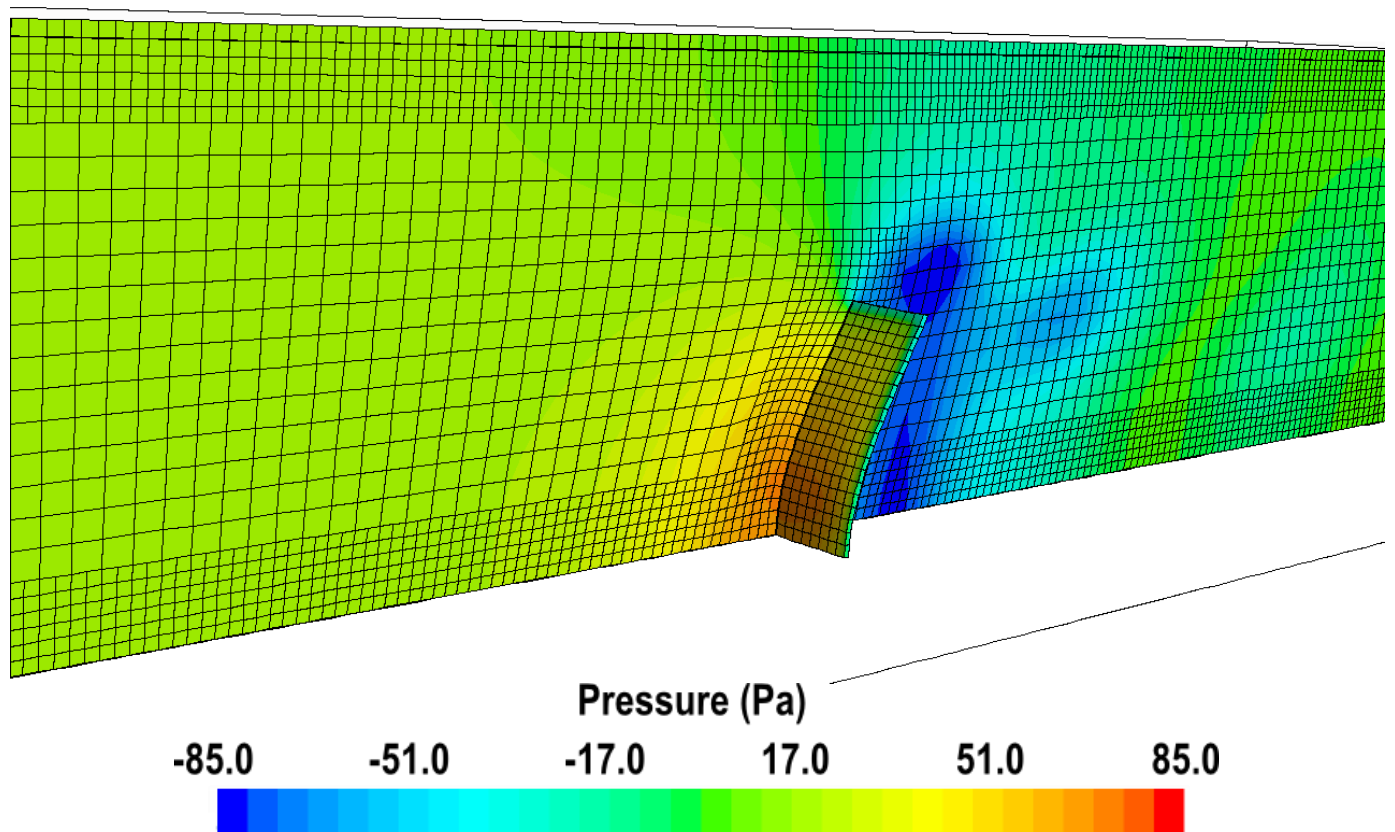

Figure 3.5: Pressure distribution in the middle plane at maximum plate deflection

Figure 3.5 presents the pressure distribution in that plane at the state of largest deformation of the plate. In the plot also the morphed mesh is displayed. Even with a coarse mesh the oscillations of the plate can be captured precisely. The mesh density and time interval between two coupling steps are dependent on the problem type and source of vibrations in the structure. In the course of the study it was concluded that depending on the behavior of the structure three ways of coupling can be defined: 
- Strong coupling - for example cable vibration where vortex shedding may play a major role in the vibration of cable. The time step between calculations needs to be small. Exchange of information between STAR-CCM+ and LS-DYNA occurs at each time step. The CFD model needs to be built carefully with great detail. Unsteady RANS may not be sufficient to model large eddy structures that may contribute to cable vibration frequency. In this case more advanced and compute intensive eddy resolving approaches may need to be considered.

- Weak coupling - for example truck rollover in the wind. Only main effects play a significant role. CFD analysis can be simplified. The time step between calculations in CFD is small but larger than in strong coupling. Exchange of information between STAR-CCM+ and LS-DYNA occurs at every 10 or so time steps.

- One way coupling - for example sign vibration due to a passing truck. Only the main effect plays a role here. The amplitude of vibration is small so there is not much need of morphing the mesh in STAR-CCM+ to the deformed shape. The pressure history is only recorded in CFD and then at the end LS-DYNA (structural) calculations are performed independently. 


\section{Weathering Steel Truck Spray Modeling and Analysis}

In the current quarter the simulation work on weathering steel truck spray modeling has finished. The results of that work were presented at the Bridge Engineering Task Force Meeting in the presentation: "Computer Modeling and Analysis of Truck Generated Salt Spray under Bridges", on August 9, 2012, in Chicago. Here a brief summary of the most recent results is presented. The results focus on explaining the influence of the traffic, wind and different geometry of approach to the bridge on the amount of salt spray particles transported to the bridge beams. In each of the considered cases a series of histograms presenting particle distribution based on their Z-position (elevation from road surface) underneath the bridge for different stages of the simulations were recorded. Subsequently cumulative plots summing up the number of particles at the bridge beam levels were created. The cumulative counts of droplets at the beam level reduce the data for each case to a single number. This data reduction allows for a simple comparison of different cases using bar graphs.

\subsection{Traffic Influence Studies}

Initially three basic cases were considered for a single passing truck with no wind: (i) an open space underneath the bridge, (ii) a sloped embankment located close to the traffic lane, and (iii) a vertical wall located $5.5 \mathrm{ft}$ from the truck. The cumulative number of particles in the bridge beam zone was calculated at three time instances: (i) in the wake of first truck, at $5.0 \mathrm{sec}$, (ii) in the wake of a second truck for simulations with two trucks, at $6.75 \mathrm{sec}$, and (iii) at the end of simulation, at $8.0 \mathrm{sec}$. The final results were plotted for the end of simulation, and the conclusions were drawn mostly on these results.

Figure 4.1 presents the number of parcels at the bridge beam level in basic cases with one and two trucks. The most parcels were transported for the case with the close vertical wall. The number of parcels for that case was over two times more than in the other cases. The influence of the close wall is non-negligible. In the case where the second truck was present, a similar tendency occurred. Comparing cases with one and two trucks the number of parcels transported to the beam level was tripled for the open and wall conditions under the bridge. For the case with close slope this value was nearly 15 times higher. The main conclusion that can be drawn from this comparison is the fact that presence of the multiple trucks closely following each other on the road can drastically increase the rate at which corrosion of bridges occurs. 


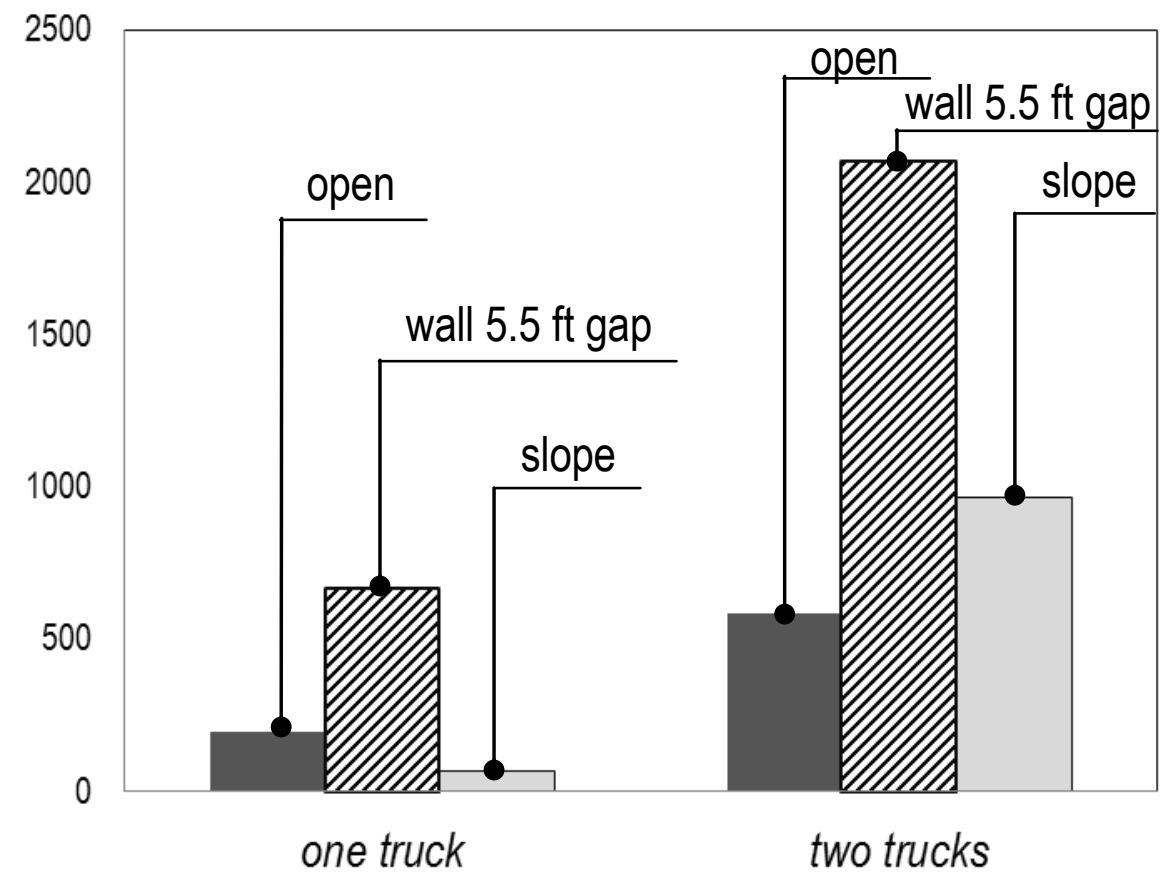

Figure 4.1: Cumulative plot presenting number of parcels at the bridge beam level in basic cases for simulations with one and two trucks

\subsection{Wind Influence Studies}

Although the wind action was not the primary parameter of this parametric study, several cases with different wind directions were included in the analysis. Only high wind of $30 \mathrm{mph}$ was considered in the analyzed cases. Four directions of the winds were simulated for the model with the sloped embankment: two from the front acting from the $45^{\circ}$ angle into the direction of motion and two from the back acting at the same $45^{\circ}$ angle. Also the wind action was studied for the case with the close vertical wall. Figure 4.2 shows schematically the direction of the winds analyzed for the case with the sloped embankment. Figure 4.3 shows the cumulative plot presenting the number of parcels at the bridge beam level for the basic cases in comparison to the cases for the sloped embankment and the wind. Even for the least severe case with the wind - with the tail wind from the right, the number of particles was nearly 20 times higher as for the base case without the wind. For the most severe case the number of parcels transported to the beam level was over 8000 , which is 120 times more than in the base case. For the cases with the wind blowing from the left side - toward the slope - the number of parcels transported to the beam level was higher. The gentle slope of the embankment was causing the flow to turn upward causing more parcels to reach the bridge beams. For the case with the close wall, this was not the situation. The parcels were either hitting the vertical wall or leaving the domain vertically up. Cases with the wind were not that severe for the vertical wall conditions under the bridge. 


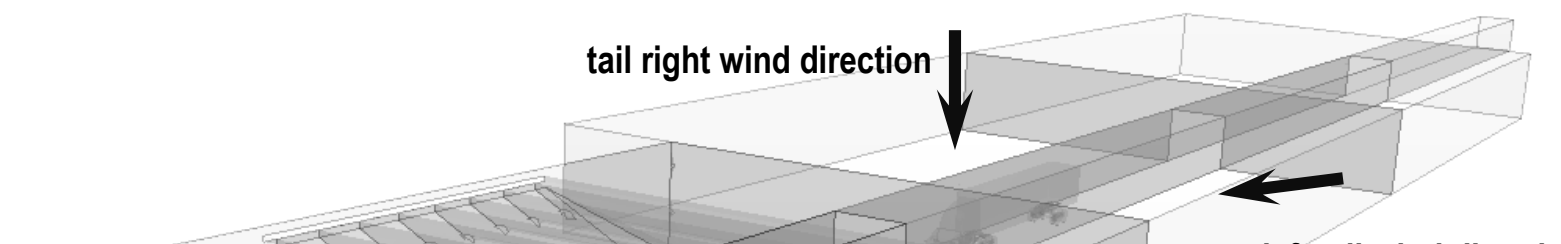

\section{Right head wind direction}

left tail wind direction

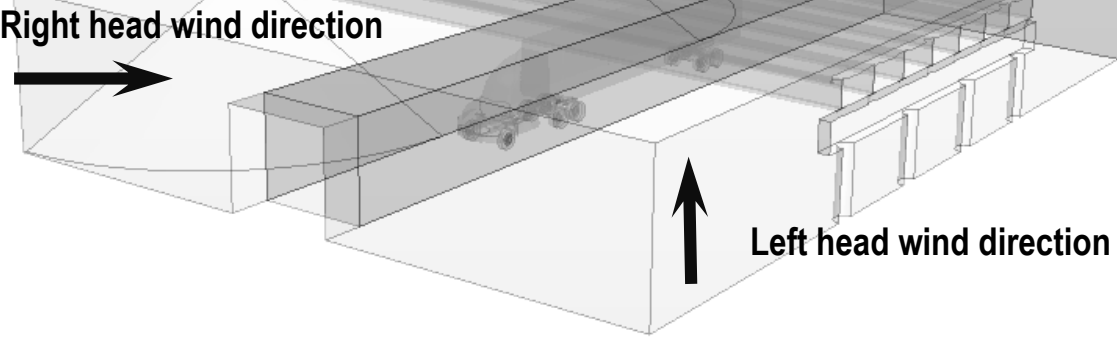

Figure 4.2: Direction of the wind analyzed for the case with sloped embankment

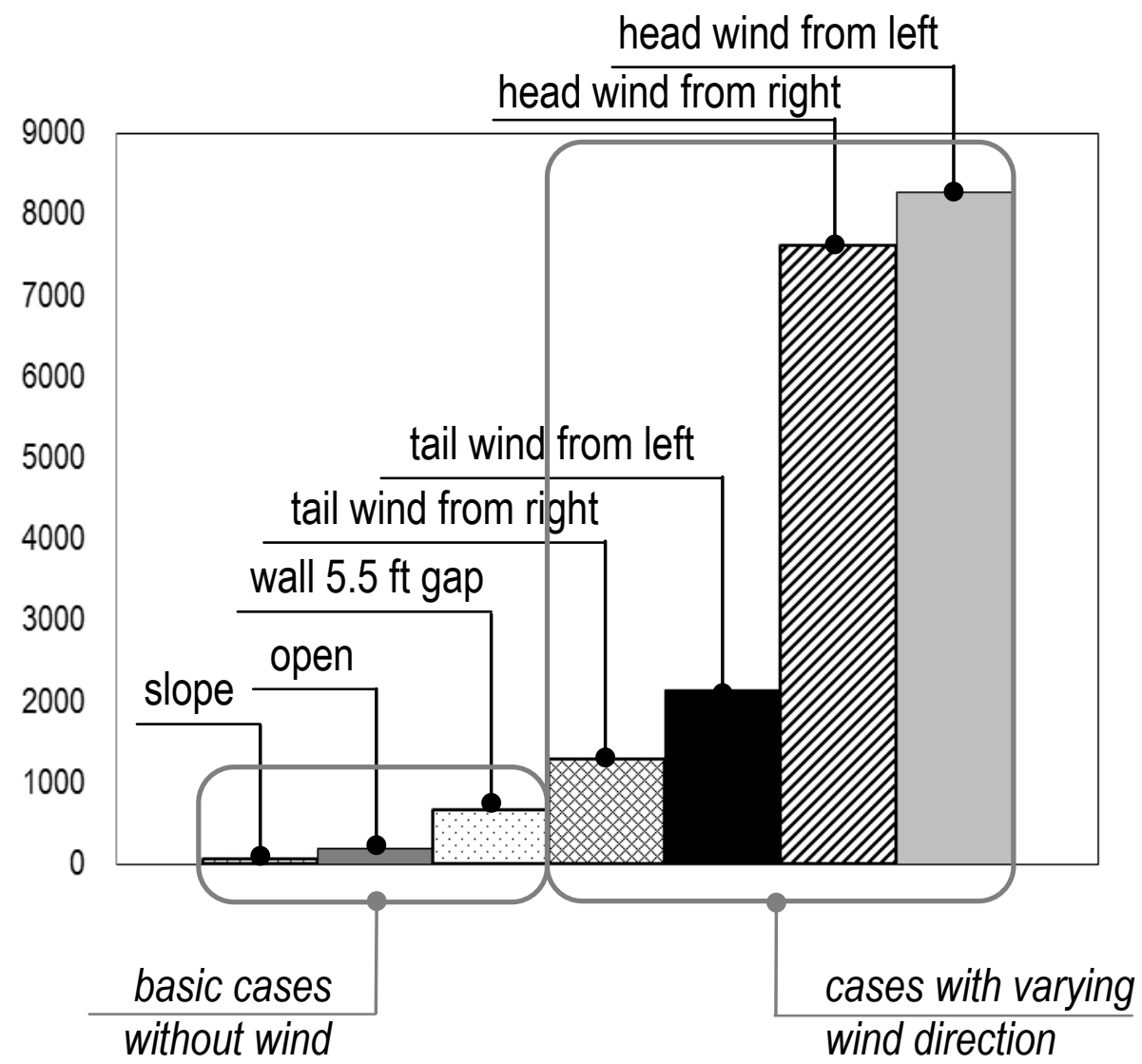

Figure 4.3: Cumulative plot presenting number of parcels at the bridge beam level for simulations with one truck and different wind conditions 


\subsection{Wall Presence Studies}

Four different location of the close short vertical wall were studied for which the wall was located at: 4 $\mathrm{ft}, 5.5 \mathrm{ft}, 9 \mathrm{ft}$ and $14 \mathrm{ft}$ from the truck. These locations are schematically shown in Figure 4.4. Besides these cases also geometries of long walls stretching through the whole computational domain were analyzed. These were called long vertical wall cases and the wall was located for these at $5.5 \mathrm{ft}$ and $14 \mathrm{ft}$ from the trailer.

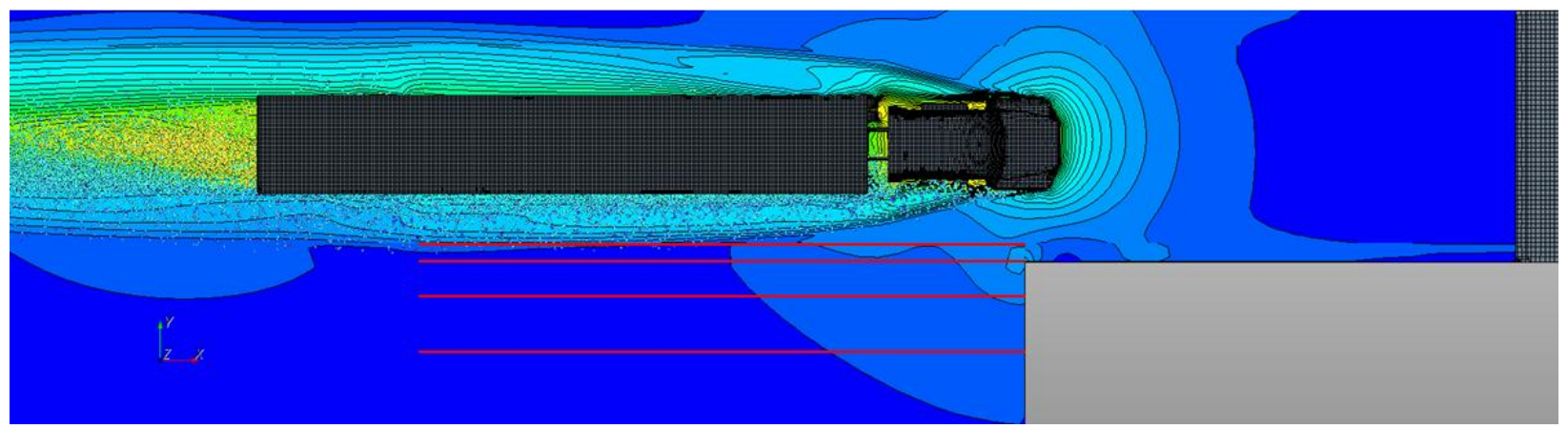

Figure 4.4: Location of the wall for tunneling effect studies

Figure 4.5 shows the cumulative plot for the basic cases together with the cases with different wall location and its vertical length. The cases with the short wall were more severe than the cases with the long wall.

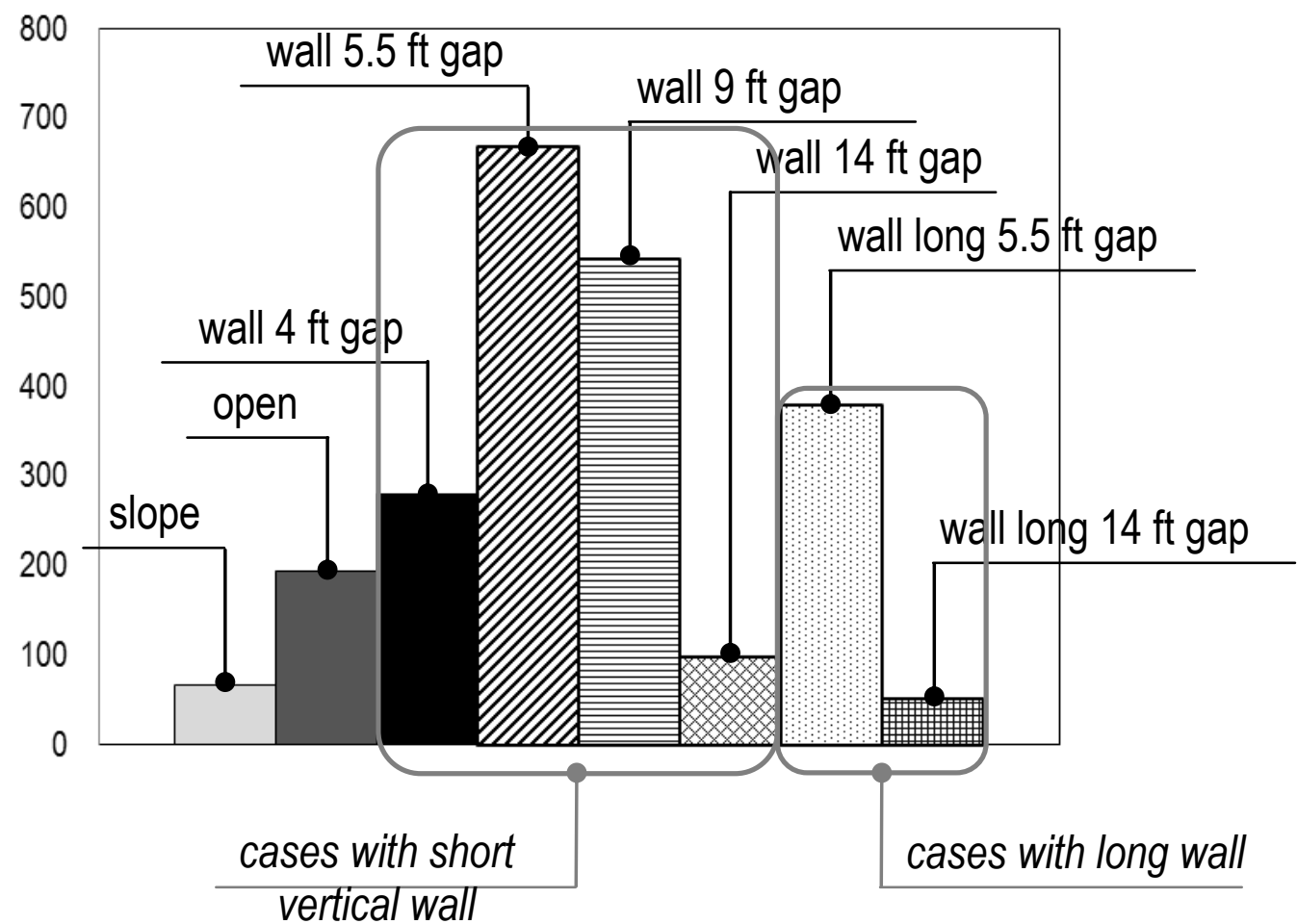

Figure 4.5: Cumulative plot presenting number of parcels at the bridge beam level for simulations with one truck and different geometry setup underneath the bridge 
The results for the short vertical wall were also extracted separately as shown in Figure 4.6. The number of parcels reaching the beam level has its maximum at the $5.5 \mathrm{ft}$ close wall distance. If possible the truck lanes shouldn't be placed next to close vertical walls to minimize the amount of salt transported to the beams.

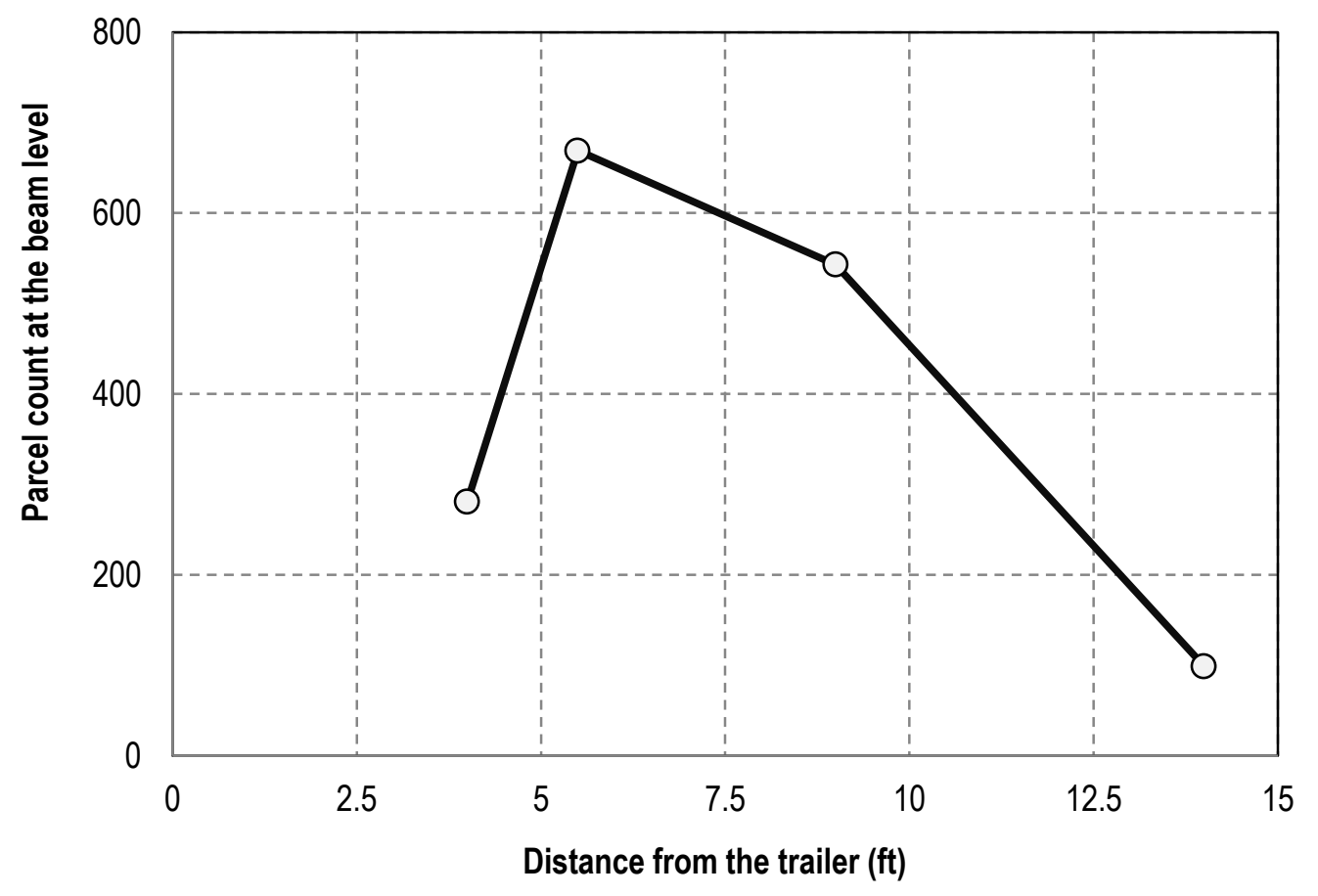

Figure 4.6: Parcel count at the bridge beam level in the models with different short vertical wall distance 


\section{Technology Transfer}

TRACC CFD staff attended the 2012 National Hydraulics Engineering Conference (NHEC) in August in Nashville, TN, and took the TRACC booth to the conference as shown in Figure 5.1. The NHEC meeting is held every other year, and is attended by a large representation of federal highway scientists and engineers, and state DOT and contractor engineers and practitioners. Four years ago there was little talk about CFD analysis at the NHEC conference in Maine beyond the appreciation of the TRACC presentation covering CFD analysis of experimental research being conducted at the TFHRC hydraulics laboratory. In addition to the TRACC presentation of current applications of CFD in analysis of scour, culverts and other problems, CFD analysis using the TRACC cluster was presented in two other presentations, and the use of CFD was mentioned favorably in a keynote talk and several other presentations. CFD was an important tool used in developing the new formula for evaluation of pressure flow sour published in the new revision of Highway Engineering Circular 18, and CFD analysis appears to be accepted by the community as an increasingly valuable tool that can be applied now to many problems and will be increasing applied in engineering research, evaluation, design, and analysis of hydraulic problems related to transportation infrastructure in the future.

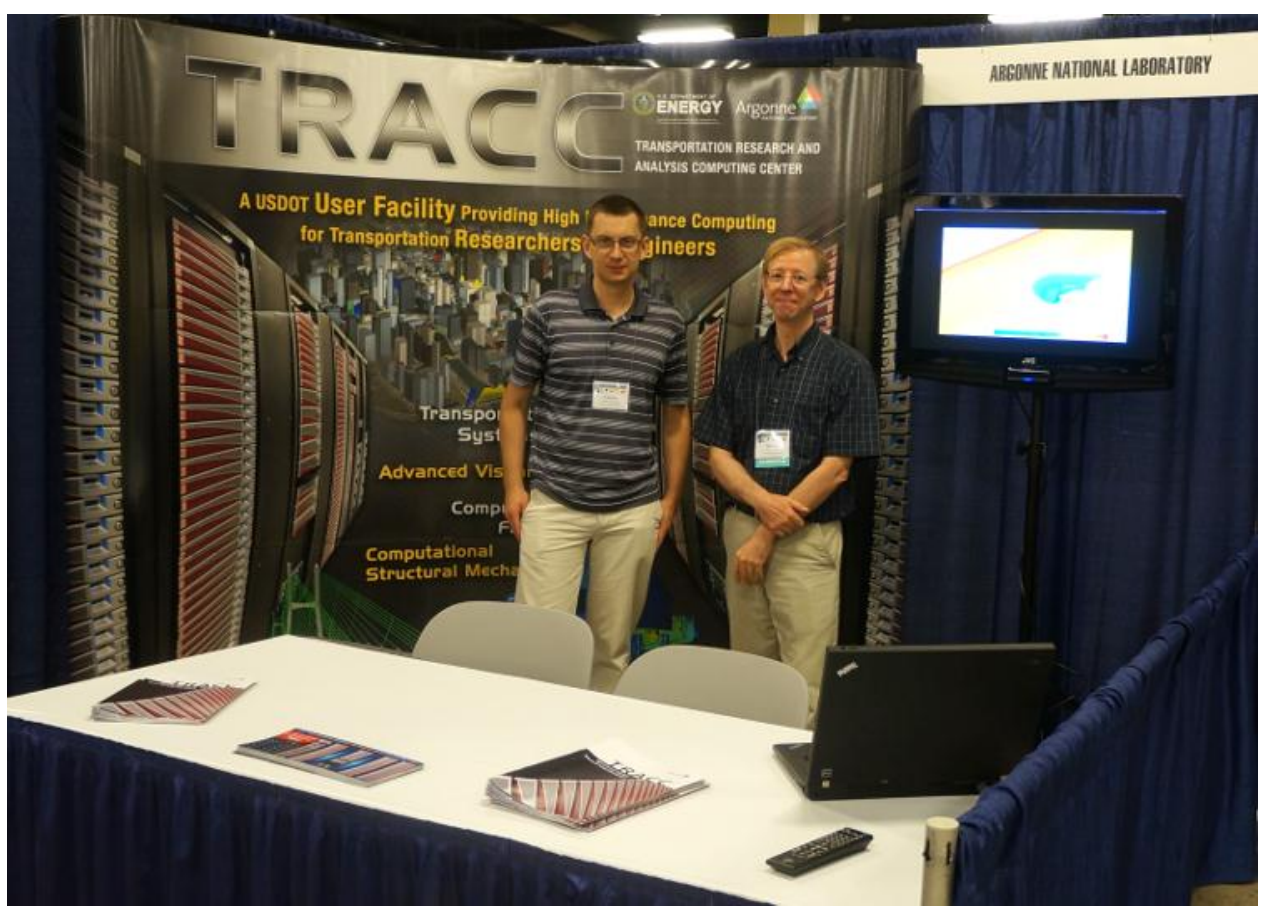

Figure 5.1 TRACC booth at the 2012 National Hydraulic Engineering Conference 


\section{TRACC Facility and User Support for TFHRC}

The original TRACC high performance computing cluster, now called the Phoenix cluster was built with 2007 hardware components and put into operation in February of 2008. At about five years old, it is still performing well, but it is aging. Its expected useful life, both computationally and economically is expected to be another 3 to 4 years. It has also been running at near saturated or saturated conditions for a large portion of its operating time. In order to provide needed high performance cluster computing resources and maintain those capabilities in a manner that will extend and expand capabilities to meet user needs for the next five years and beyond, a new TRACC cluster was acquired over the course of the past year. The new cluster, named Zephyr from Atipa systems, more than doubles TRACC's computing capacity with up to date hardware and Infiniband connectivity that is twice as fast as that used in the Phoenix cluster (40 Gbit/s in Zephyr versus $20 \mathrm{Gbit} / \mathrm{s}$ in Phoenix).

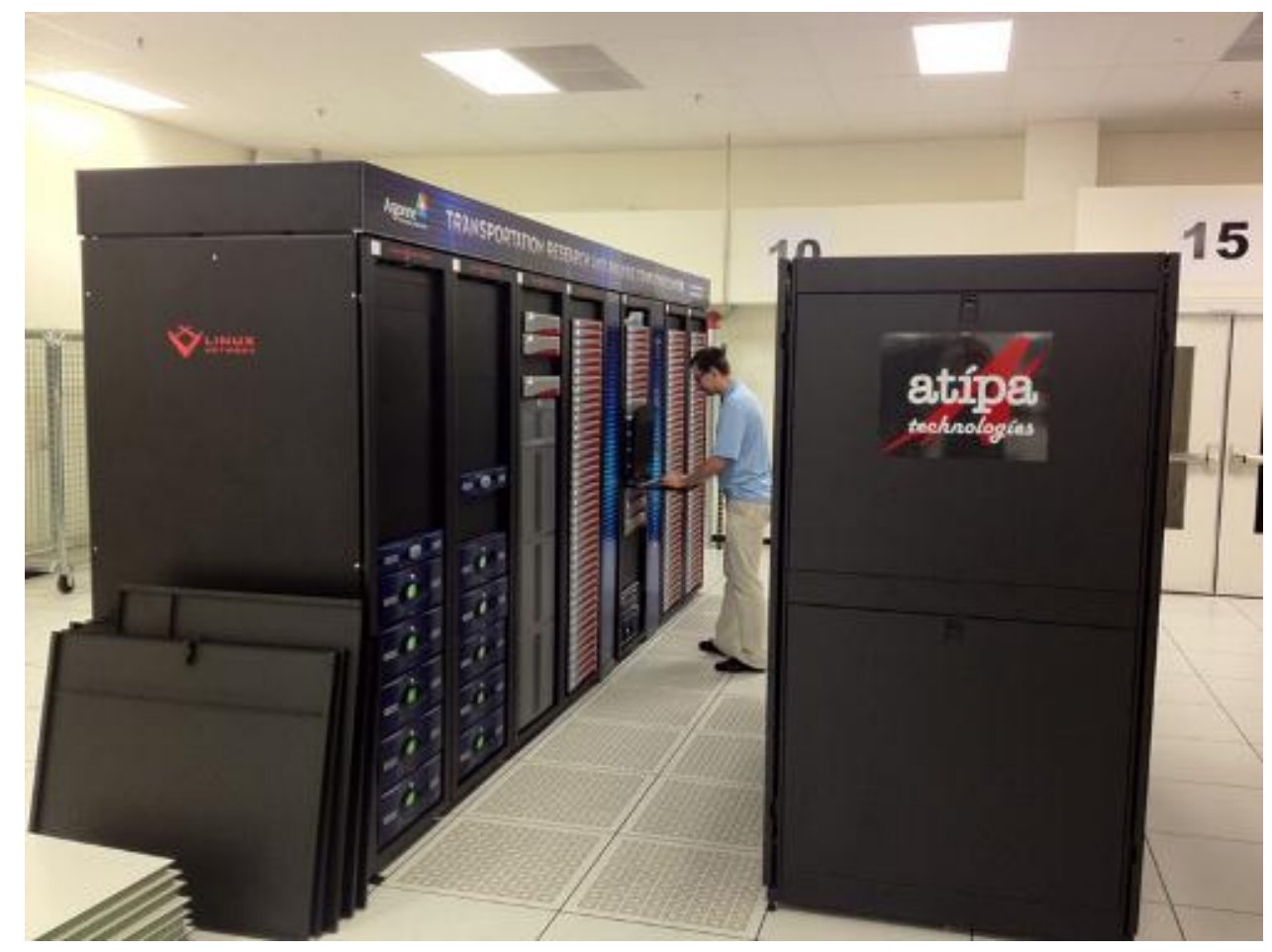

Figure 6.1 TRACC Phoenix cluster (left) and Zephyr cluster (right) in the machine room at Argonne's high performance computing facility.

Each of the 88 regular compute nodes on the Zephyr cluster has 4 times the memory and twice as many floating point processors, allowing each compute node to handle two to four times the work of a compute node on the Phoenix cluster. In addition the Zephyr cluster has two 64 GB memory nodes and two 128 GB nodes for jobs that require large amounts, such as mesh generation for very large problems.

More information on the Zephyr cluster will be available on the TRACC web site (www.tracc.anl.gov), and detailed information on the procedures for using Zephyr and any differences in procedures between Zephyr and Phoenix are being documented on the TRACC wiki: (https://wiki.anl.gov/tracc/Main Page). 


\subsection{Zephyr Cluster Configuration}

- 92 computing nodes each with two AMD Interlagos 16 integer, 8 floading point core, $2.3 \mathrm{GHz}$ processors ( 32 integer cores, 16 floating point cores) per node, 2944 cores total)

- Arranged in three queues

- 88 nodes with $32 \mathrm{~GB}$ of memory (1GB per core)

- 2 nodes with $64 \mathrm{~GB}$ of memory (2GB per core)

- 2 nodes with $128 \mathrm{~GB}$ of memory (4GB per core)

- 120 TB of Lustre-based user storage in a RAID6 configuration

- High-speed 108 port fat-free QDR, 40 Gbit/s Infiniband connectivity

- 240 ports of Gigabit Ethernet connectivity

- Dual 10 Gbps connectivity to Argonne and external networks

- Interconnectivity between the new cluster (Zephyr) and the old cluster (Phoenix)

- Two administrative servers

- One I/O server

- One monitoring and statistics gathering server

- One application server that can be used for user code development (sandbox)

- All nodes run Linux using CentOS 6.2

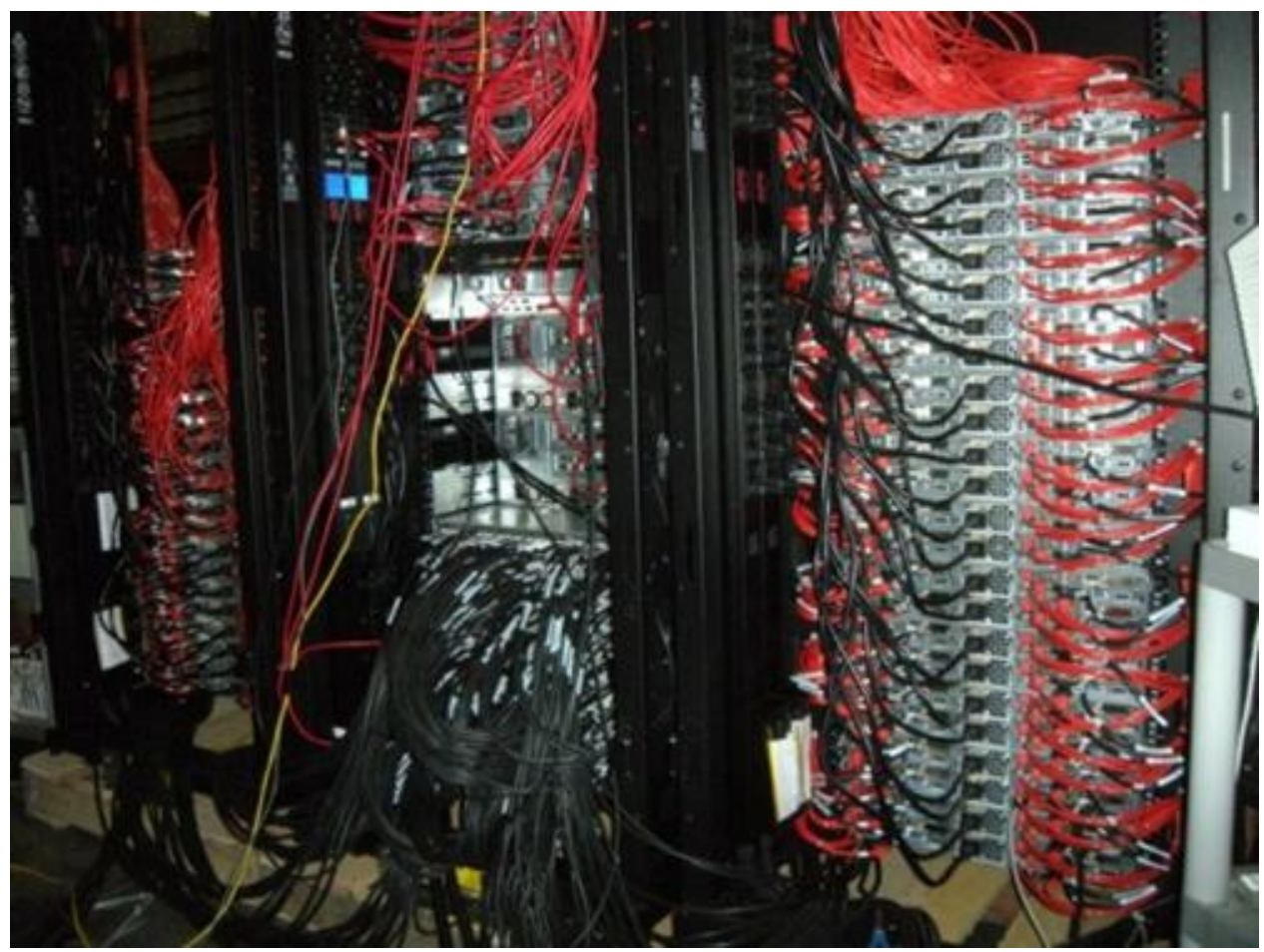

Figure 6.2 Zephyr backplane showing dense cabling due to the dense configuration of 4 compute nodes per $2 \mathrm{U}$ rack in the cabinets. 


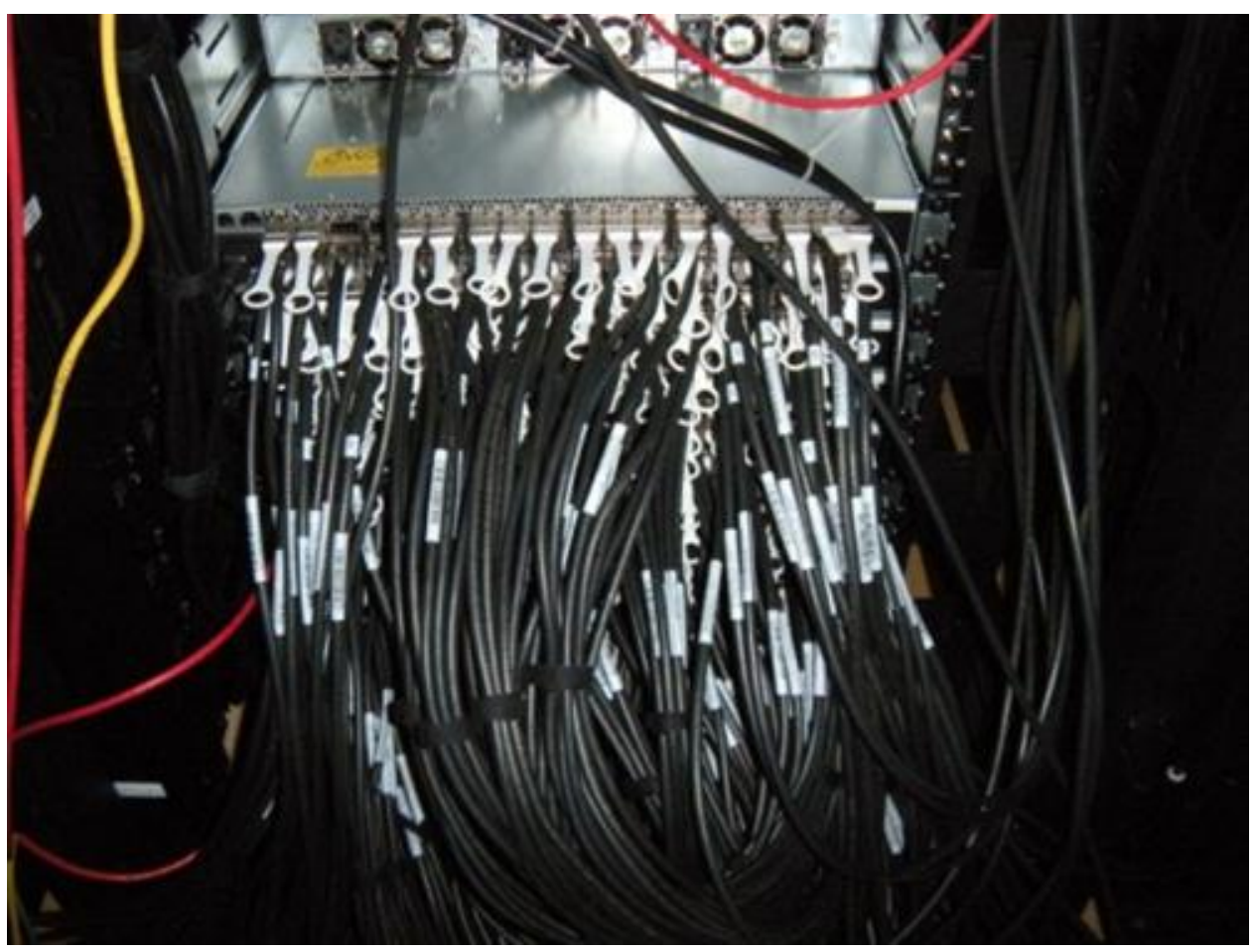

Figure 6.3 High density of cables at the $40 \mathrm{~GB} / \mathrm{s}$ Infiniband interconnect switches between cluster nodes

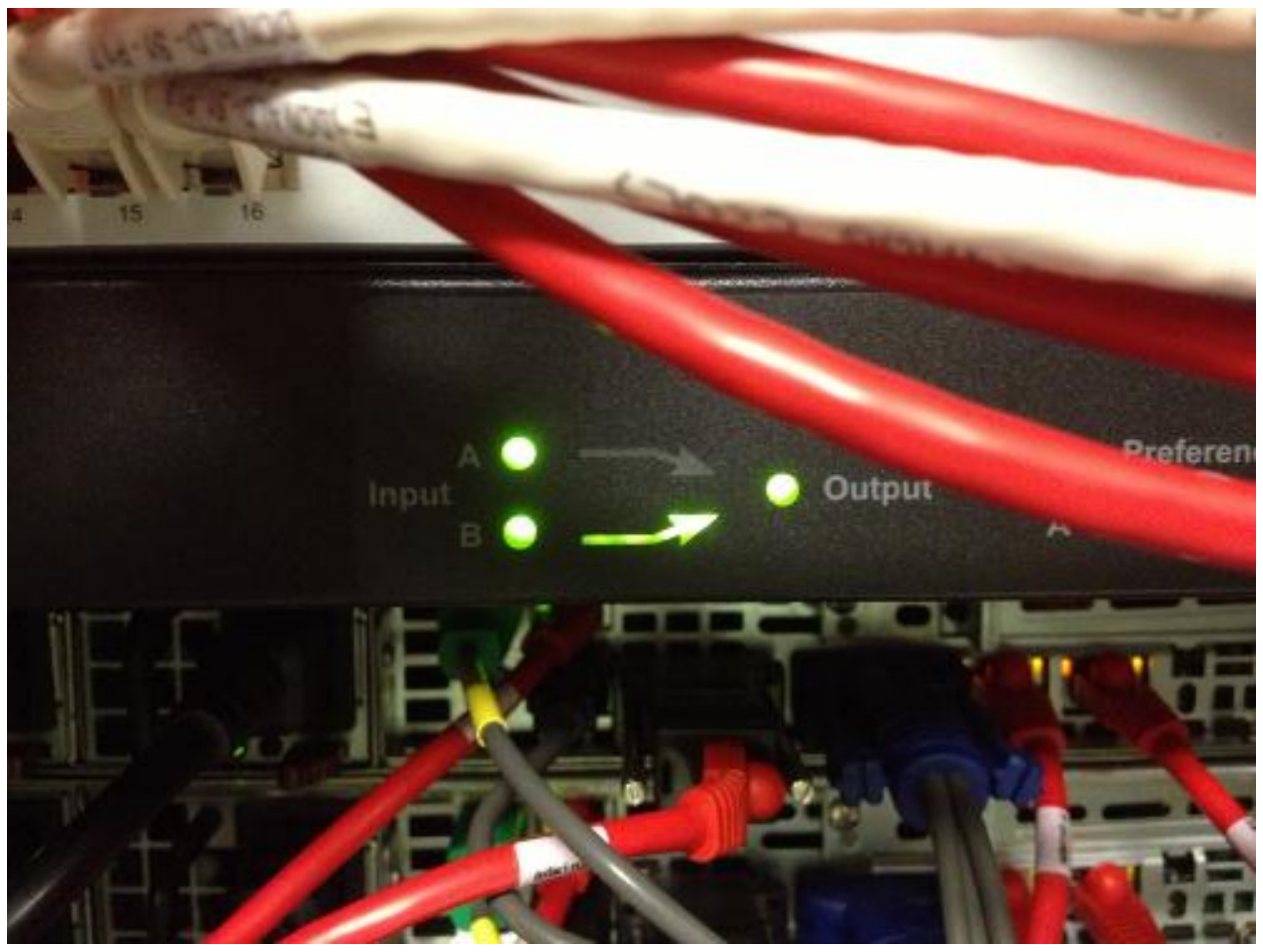

Figure 6.4 The Zephyr cluster is powered from two independent circuits. An automatic failover switch provides power to cluster components with a single power supply if one of the supply circuits is interrupted. 


\section{Argonne}

\section{Energy Systems Division}

Argonne National Laboratory

9700 South Cass Avenue, Bldg. 362

Argonne, IL 60439-4815

www.anl.gov

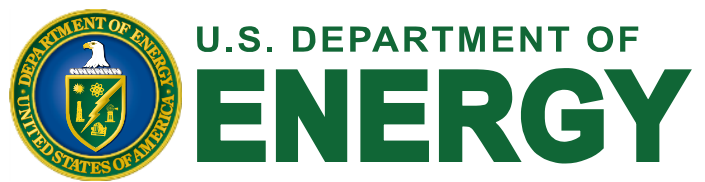

Argonne National Laboratory is a U.S. Department of Energy laboratory managed by UChicago Argonne, LLC 